\title{
Large time asymptotic behavior of the compressible Navier-Stokes Equations in partial Space-Periodic Domains
}

\author{
Yin Huicheng*, Zhang Lin*, Zhu Lu* \\ (Department of Mathematics and IMS, Nanjing University, Nanjing 210093, China.)
}

\begin{abstract}
In this paper, we study the large time behavior of the 3-D isentropic compressible NavierStokes equation in the partial space-periodic domains, and simultaneously show that the related profile systems can be described by like Navier-Stokes equations with suitable "pressure" functions in lower dimensions. Our proofs are based on the energy methods together with some delicate analysis on the corresponding linearized problems.
\end{abstract}

Keywords: Large time behavior, profile system, energy method, partial space-periodic domain, Fourier series

Mathematical Subject Classification 2000: 35Q30, 76N10

\section{$\S 1$. Introduction and main results}

In this paper, we consider the 3-D isentropic compressible Navier-Stokes equation for $(t, z) \in$ $[0,+\infty) \times \Omega$ :

$$
\left\{\begin{array}{l}
\partial_{t} \rho+\operatorname{div} m=0, \\
\partial_{t} m+\operatorname{div}\left(\frac{m \otimes m}{\rho}\right)+\nabla p(\rho)=\mu \Delta\left(\frac{m}{\rho}\right)+\left(\mu+\mu^{\prime}\right) \nabla \operatorname{div}\left(\frac{m}{\rho}\right),
\end{array}\right.
$$

where $\Omega=\mathbb{T}^{\ell} \times \mathbb{R}^{3-\ell}, \mathbb{T}^{\ell}=[0,2 \pi]^{\ell}$ is the $\ell$-dimensional torus $(1 \leq \ell \leq 3), z=\left(z_{1}, z_{2}, z_{3}\right)$, $\rho=\rho(t, z)$ is the density, $m=\left(m^{1}, m^{2}, m^{3}\right)(t, z)$ is the momentum, $p=p(\rho)$ is the pressure, $\mu$ and $\mu^{\prime}$ are the first and the second viscosity coefficient respectively, which satisfy $\mu>0$ and

${ }^{*}$ This project was supported by the NSFC ( No. 11025105), and by the Priority Academic Program Development of Jiangsu Higher Education Institutions. 
$\frac{2}{3} \mu+\mu^{\prime} \geq 0$. The initial data of (1.1) are given as follows

$$
(\rho, m)(0, z)=\left(1+\rho_{0}(z), m_{0}(z)\right)
$$

where $\left(\rho_{0}, m_{0}\right) \in H^{4}(\Omega) \times\left(H^{4}(\Omega)\right)^{3}$, and $1+\rho_{0}(z)>0$ for $z \in \Omega$.

It is obvious that $(\rho, m)=(1,0)$ is a steady solution of $(1.1)$ with the initial data $(\rho, m)(0, z)$ $=(1,0)$. We will be concerned with the perturbation problem of (1.1) to this constant state. Denote by

$$
\nu_{1}=\mu, \nu_{2}=\mu+\mu^{\prime}, \gamma=\sqrt{p^{\prime}(1)}
$$

As in $[14,15]$, if we set $\phi=\gamma(\rho-1)$, then (1.1)-(1.2) can be rewritten as

$$
\left\{\begin{array}{l}
\partial_{t} \phi+\gamma \operatorname{div} m=0 \\
\partial_{t} m-\nu_{1} \Delta m-\nu_{2} \nabla \operatorname{div} m+\gamma \nabla \phi=G(\phi, m), \\
(\phi, m)(0, z)=\left(\phi_{0}, m_{0}\right)(z)
\end{array}\right.
$$

where $\phi_{0}(z)=\gamma \rho_{0}(z)$ and

$$
\begin{aligned}
G(\phi, m)= & -\operatorname{div}\left(\frac{\gamma}{\phi+\gamma} m \otimes m\right)-\nu_{1} \Delta\left(\frac{\phi}{\phi+\gamma} m\right)-\nu_{2} \nabla \operatorname{div}\left(\frac{\phi}{\phi+\gamma} m\right) \\
& -\nabla\left\{\frac{\phi^{2}}{\gamma^{2}} \int_{0}^{1}(1-\theta)^{2} p^{\prime \prime}\left(1+\frac{\theta \phi}{\gamma}\right) d \theta\right\} .
\end{aligned}
$$

We now introduce some notations for later uses. The Fourier transformation of the function $f \in L^{1}\left(\mathbb{T}^{\ell} \times \mathbb{R}^{3-\ell}\right)$ is denoted by

$$
\mathcal{F}(f)(k, \xi)=\hat{f}(k, \xi)=\int_{\mathbb{T}^{\ell}} \int_{\mathbb{R}^{3-\ell}} e^{-i(k \cdot x+\xi \cdot y)} f(x, y) d x d y
$$

where $z=(x, y), x=\left(z_{1}, \cdots, z_{l}\right) \in \mathbb{T}^{\ell}, y=\left(y_{1}, \cdots, y_{3-\ell}\right)=\left(z_{\ell+1}, \cdots, z_{3}\right) \in \mathbb{R}^{3-\ell}, k=$ $\left(k_{1}, \cdots, k_{\ell}\right) \in \mathbb{Z}^{\ell}, \xi=\left(\xi_{1}, \cdots, \xi_{3-\ell}\right) \in \mathbb{R}^{3-\ell}$. The inverse Fourier transformation of the sequence $\{g(k, \xi)\}_{k \in \mathbb{Z}^{l}}$ is defined as

$$
\left(\mathcal{F}^{-1} g\right)(z)=\frac{1}{(2 \pi)^{\ell}} \sum_{k \in \mathbb{Z}^{\ell}} e^{i k \cdot x} \int_{\mathbb{R}^{3-\ell}} e^{i \xi \cdot y} g(k, \xi) d \xi .
$$

Write the mean value of $f(z)$ over $\mathbb{T}^{\ell}$ as $\bar{f}(y)$ :

$$
\bar{f}(y)=\frac{1}{(2 \pi)^{\ell}} \int_{\mathbb{T}^{\ell}} f(x, y) d x .
$$

In addition, we define

$$
\operatorname{div}^{\prime} v=\partial_{y_{1}} v_{1}+\cdots+\partial_{y_{3-\ell}} v_{3-\ell}, \nabla^{\prime}=\left(\partial_{y_{1}}, \cdots, \partial_{y_{3-\ell}}\right)^{T}, \Delta^{\prime}=\partial_{y_{1}}^{2}+\cdots+\partial_{y_{3-\ell}}^{2},
$$

where $v=\left(v_{1}, \cdots, v_{3-\ell}\right)^{T}$. And we denote $\|u(t, \cdot)\|_{L^{p}(\Omega)}$ as $\|u\|_{L^{p}}$ for $1 \leq p \leq \infty$. 
For different $\ell$, our main results in this paper are

Theorem 1.1. For $\ell=1$, if $u_{0} \in H^{4}\left(\mathbb{T} \times \mathbb{R}^{2}\right)$ and $\left\|u_{0}\right\|_{H^{4} \cap L^{1}} \leq \varepsilon$, then for small $\varepsilon>0$, (1.3) has a global solution $u(t, z)=(\phi, m)(t, z) \in C\left([0,+\infty), H^{4}\left(\mathbb{T} \times \mathbb{R}^{2}\right)\right) \cap C^{1}\left([0,+\infty), H^{2}(\mathbb{T} \times\right.$ $\left.\mathbb{R}^{2}\right)$ ) satisfying for $t \rightarrow+\infty$

$$
\begin{aligned}
& \left\|\partial_{z}^{k} u\right\|_{L^{2}}=O\left(t^{-\frac{1}{2}-\frac{k}{2}}\right), \quad k=0,1, \\
& \left\|\partial_{z}^{k} u\right\|_{L^{2}}=O\left(t^{-\frac{4-k}{3}}\right), \quad k=2,3,4, \\
& \|u(t, z)-\eta(t, y)\|_{L^{2}}=O\left(t^{-1}\right),
\end{aligned}
$$

where the profile $\eta(t, y)=(\sigma, w)(t, y)$ satisfies the following system

$$
\left\{\begin{array}{l}
\partial_{t} \sigma+\gamma d i v^{\prime} w^{\prime}=0 \\
\partial_{t} w_{1}-\nu_{1} \Delta^{\prime} w_{1}=-\operatorname{div}^{\prime}\left(w_{1} w^{\prime}\right) \\
\partial_{t} w^{\prime}-\nu_{1} \Delta^{\prime} w^{\prime}-\nu_{2} \nabla^{\prime} d i v^{\prime} w^{\prime}+\gamma \nabla^{\prime} \sigma=-\operatorname{div}^{\prime}\left(w^{\prime} \otimes w^{\prime}\right)-\alpha \nabla^{\prime}\left(\sigma^{2}\right) \\
(\sigma, w)(0, y)=\left(\bar{\phi}_{0}, \bar{m}_{0}\right)(y)
\end{array}\right.
$$

here and below $\alpha=\frac{p^{\prime \prime}(1)}{2 \gamma^{2}}>0, w^{\prime}=\left(w_{2}, w_{3}\right)$.

Theorem 1.2. For $\ell=2$, if $u_{0} \in H^{4}\left(\mathbb{T}^{2} \times \mathbb{R}\right)$ and $\left\|u_{0}\right\|_{H^{4} \cap L^{1}} \leq \varepsilon$, then for small $\varepsilon>0$, (1.3) has a global solution $u(t, z)=(\phi, m)(t, z) \in C\left([0,+\infty), H^{4}\left(\mathbb{T}^{2} \times \mathbb{R}\right)\right) \cap C^{1}\left([0,+\infty), H^{2}\left(\mathbb{T}^{2} \times\right.\right.$ $\mathbb{R})$ ) satisfing for $t \rightarrow+\infty$

$$
\begin{aligned}
& \left\|\partial_{z}^{k} u\right\|_{L^{2}}=O\left(t^{-\frac{1}{4}-\frac{k}{2}}\right), \quad k=0,1, \\
& \left\|\partial_{z}^{k} u\right\|_{L^{2}}=O\left(t^{-\frac{4-k}{4}}\right), \quad k=2,3,4 \\
& \|u(t, z)-\eta(t, y)\|_{L^{2}}=O\left(t^{-\frac{3}{4}+\delta}\right), \quad \forall \delta>0
\end{aligned}
$$

where $\eta(t, y)=(\sigma, w)(t, y)$ satisfies the following equations

$$
\left\{\begin{array}{l}
\partial_{t} \sigma+\gamma \partial_{y} w_{3}=0 \\
\partial_{t} w_{j}-\nu_{1} \partial_{y}^{2} w_{j}=-\partial_{y}\left(w_{i} w_{3}\right), j=1,2 \\
\partial_{t} w_{3}-\left(\nu_{1}+\nu_{2}\right) \partial_{y}^{2} w_{3}+\gamma \partial_{y} \sigma=-\partial_{y} w_{3}^{2}-\alpha \partial_{y} \sigma^{2} \\
(\sigma, w)(0, y)=\left(\bar{\phi}_{0}, \bar{m}_{0}\right)(y)
\end{array}\right.
$$

Theorem 1.3. For $\ell=3$, if $u_{0} \in H^{4}\left(\mathbb{T}^{3}\right)$ and $\left\|u_{0}\right\|_{H^{4}} \leq \varepsilon$, then for small $\varepsilon>0$, (1.3) has a global solution $u(t, z)=(\phi, m)(t, z) \in C\left([0,+\infty), H^{4}\left(\mathbb{T}^{3}\right)\right) \cap C^{1}\left([0,+\infty), H^{2}\left(\mathbb{T}^{3}\right)\right)$ satisfying $\left\|u-\bar{u}_{0}\right\|_{H^{1}} \leq C e^{-a_{0} t}\left\|u_{0}\right\|_{H^{4}}$, where $a_{0}>0$ is some determined number.

Remark 1.1. The local existence and uniqueness of the solution to the problem (1.3) have been shown in [16]. In addition, the global well-posedness of (1.3) was obtained by the Matsumura-Nishida energy method in [13-15]. So the purpose of this paper is to study the large-time behavior of the solution to (1.3) and find the corresponding profile systems. 
Remark 1.2. For the general $N$-dimensional $(N \geq 4)$ isentropic compressible NavierStokes equation (1.3), by the same analysis as in Theorem 1.1-Theorem 1.3, we can get the following conclusions: Assuming $u_{0}=\left(\phi_{0}, m_{0}\right) \in H^{s}\left(\mathbb{T}^{\ell} \times \mathbb{R}^{N-\ell}\right)$ and $\left\|u_{0}\right\|_{H^{s} \cap L^{1}} \leq \varepsilon$, where $s \geq\left[\frac{N}{2}\right]+3,1 \leq \ell \leq N-1$, and $\varepsilon>0$ is small enough, then

$$
\begin{aligned}
& \left\|\partial_{z}^{k} u\right\|_{L^{2}}=O\left(t^{-\frac{N-l}{4}-\frac{k}{2}}\right), \quad k=0,1, \\
& \|u(t, z)-\eta(t, y)\|_{L^{2}}=O\left(t^{-\frac{N-\ell+2}{4}}\right),
\end{aligned}
$$

where $u=(\phi, m)$, and the profile $\eta(t, y)=(\sigma, w)(t, y)$ satisfies the following partial differential equation system

$$
\left\{\begin{array}{l}
\partial_{t} \sigma+\gamma d i v^{\prime} V=0 \\
\partial_{t} U-\nu_{1} \Delta^{\prime} U=-\operatorname{div}^{\prime}(V \otimes U) \\
\partial_{t} V-\nu_{1} \Delta^{\prime} V-\nu_{2} \nabla^{\prime} \operatorname{div}^{\prime} V+\gamma \nabla^{\prime} \sigma=-\operatorname{div}^{\prime}(V \otimes V)-\alpha \nabla^{\prime}\left(\sigma^{2}\right) \\
(\sigma, w)(0, y)=\left(\bar{\phi}_{0}, \bar{m}_{0}\right)(y)
\end{array}\right.
$$

here $w=(U, V), U=\left(w_{1}, \ldots, w_{\ell}\right)$ and $V=\left(w_{\ell+1}, \ldots, w_{N}\right)$.

If $\ell=N$, the exponential decay property $\left\|u-\bar{u}_{0}\right\|_{H^{1}} \leq C e^{-a_{0} t}\left\|u_{0}\right\|_{H^{4}}$ as in Theorem 1.3 also holds.

Remark 1.3. In Theorem 1.1, the decay property (1.6) comes from the integral

$$
\int_{0}^{t}(1+t-\tau)^{-1}(1+\tau)^{-\frac{3}{2}} d \tau=O\left(t^{-1}\right)
$$

In Theorem 1.2, the decay property (1.10) comes from the integral

$$
\int_{0}^{t}(1+t-\tau)^{-\frac{3}{4}}(1+\tau)^{-1} d \tau=O\left(t^{-\frac{3}{4}} \ln t\right)=O\left(t^{-\frac{3}{4}+\delta}\right) \quad \text { for any } \delta>0 .
$$

One can find more details on the proofs of (1.6) and (1.10) in $\S 5$ and $\S 6$ below, respectively.

In addition, the estimates (1.5) and (1.9) are obtained by the interpolation between the uniform boundedness of $\|u\|_{C\left([0,+\infty), H^{4}\right)}$ and the decay estimates of the first-order derivatives in (1.4) and (1.8), respectively.

We now mention some interesting or remarkable results which are closely related to our works. In [6-9], the authors studied the large time behavior of small perturbed solutions to the compressible isentropic Navier-Stokes equations in the infinite cylindrical domains or parallel domains with Dirichlet boundary conditions of the velocity. For the initial value problem in the whole space, the optimal decay properties of global small perturbed solutions have been established in [2-6], [11-13] and the references therein. With respect to the initial-boundary value problem, the optimal decay property have also been obtained in [10], [14-15] and so on.

Next we comment on the proofs of Theorem 1.1-Theorem 1.3. In [8-9], under the smallness assumptions on the Reynolds number, the Mach number and the initial perturbation, the authors obtain the large time behavior of the solution and show that the related profile system 
is described by the 1-D viscous Burgers equation. Moreover, it is proved in [8-9] that the decay properties of the solution are determined by the lower-energy part meanwhile the asymptotic behavior depends on the first simple zero eigenvalue of the resulting linearized operator. However, in our cases, there are some differences from those in [8-9]. The first difference is: due to the influences of the partial periodic variables in (1.3), we have to use the Fourier series and Fourier transformation simultaneously to analyze the linearized equation of (1.3), and which yields that the first zero eigenvalue of the linearized equation in the low frequency is of four multiplicities, while the zero-eigenvalue is simple and only Fourier transformation is applied in [8-9]. This leads to that we should consider the difference of the solution $u$ and its mean value $\bar{u}$ on the periodic variables (fortunately, $\bar{u}$ is just only the right eigenvector of zero eigenvalue) in order to obtain more precise decay property. Here we point out that only the difference of the density $\rho$ and its mean value $\bar{\rho}$ on the cross section needs to be considered in [8-9]. The second difference is: the resulting profile systems (1.7) and (1.11) are the nonlinear partial differential systems other than the scalar viscous Burgers equation as in [8-9]. Thanks to some delicate analysis on the linearized system together with a variant of the Matrumura-Nishida energy method, we can complete the proofs of Theorem 1.1-Theorem 1.3.

The rest of the paper is organized as follows: In $\S 2$, we will establish some large time properties on the solution to the homogeneous linearized problem of (1.3). In $\S 3$, we study the well-posedness and the asymptotic behavior of the solution to the auxiliary problem (1.7). In $\S 4$ and $\S 5$, we complete the proof of Theorem 1.1. Finally, Theorem 1.2 and Theorem 1.3 are proved in $\S 6$.

\section{§2. Some analysis on the homogeneous linearized problem of (1.3)}

In this section, we will study the properties of the solution to the homogeneous linearized problem of (1.3). Namely, we consider the following linear problem

$$
\left\{\begin{array}{l}
\partial_{t} v+L v=0 \\
v(0, z)=u_{0}(z)
\end{array}\right.
$$

where $v=(\phi, m), u_{0}=\left(\phi_{0}, m_{0}\right)$, and $L=\left(\begin{array}{cc}0 & \gamma \operatorname{div} \\ \gamma \nabla & -\nu_{1} \Delta I_{3}-\nu_{2} \nabla \operatorname{div}\end{array}\right)$ with $I_{3}$ being the $3 \times 3$ identity matrix.

Taking both the Fourier transformation on the $y$ variables and the Fourier series expansion on the $x$ variables in (2.1), we have

$$
\left\{\begin{array}{l}
\frac{d}{d t}\left(\begin{array}{c}
\hat{\phi}(t, k, \xi) \\
\hat{m}(t, k, \xi)
\end{array}\right)+\hat{L}(k, \xi)\left(\begin{array}{c}
\hat{\phi}(t, k, \xi) \\
\hat{m}(t, k, \xi)
\end{array}\right)=0, \\
(\hat{\phi}, \hat{m})(0, k, \xi)=\left(\hat{\phi}_{0}, \hat{m}_{0}\right)(k, \xi)
\end{array}\right.
$$

where $k \in \mathbb{Z}^{\ell}, \xi \in \mathbb{R}^{3-\ell}$, and

$$
\hat{L}(k, \xi)=\left(\begin{array}{ccc}
0 & i \gamma k & i \gamma \xi \\
i \gamma k^{T} & \nu_{1}\left(|k|^{2}+\xi^{2}\right)+\nu_{2} k^{T} k & \nu_{2} k^{T} \xi \\
i \gamma \xi^{T} & \nu_{2} \xi k^{T} & \left(\nu_{1}|k|^{2}+\left(\nu_{1}+\nu_{2}\right)|\xi|^{2}\right) I_{2}
\end{array}\right) .
$$


It follows from a direct computation that the eigenvalues of $\hat{L}(k, \xi)$ are

$$
\lambda_{1}=\lambda_{2}=\nu_{1} p, \lambda_{ \pm}=\frac{1}{2}\left\{\left(\nu_{1}+\nu_{2}\right) p \pm \sqrt{\left(\nu_{1}+\nu_{2}\right)^{2} p^{2}-4 \gamma^{2} p}\right\}
$$

where $p=|k|^{2}+|\xi|^{2}$. Obviously, $\lambda_{1}=\lambda_{2}=\lambda_{ \pm}=0$ for $p=0$, which means that 0 is the 4 root of $\hat{L}(k, \xi)$ as $p=0$. In addition, $\lambda_{1}$ and $\lambda_{2}$ are positive numbers for $p>0, \lambda_{ \pm}$are complex numbers when $p>0$ is small, and $\lambda_{+} \rightarrow+\infty, \lambda_{-} \rightarrow \frac{\gamma^{2}}{\nu_{1}+\nu_{2}}$ as $p \rightarrow+\infty$. Therefore, if we choose a fixed positive number $r_{0}$ with $0<r_{0}^{2}<\min \left\{1, \frac{\gamma^{2}}{\left(\nu_{1}+\nu_{2}\right)^{2}}\right\}$, then we can conclude that for $p \geq r_{0}^{2}$

$$
\max \left\{\lambda_{1}, \lambda_{2}, \operatorname{Re} \lambda_{-}, \operatorname{Re} \lambda_{+}\right\} \geq \min \left\{\nu_{1} r_{0}^{2}, \frac{\gamma^{2}}{2\left(\nu_{1}+\nu_{2}\right)}\right\} \triangleq a>0 .
$$

From this, one can check that the solution to (2.1) can be written as

$$
v(t, z)=\mathcal{U}(t) u_{0} \triangleq\left(e^{-t L} u_{0}\right)(t, z)=\frac{1}{(2 \pi)^{\ell}} \sum_{k \in \mathbb{Z}^{l}} e^{i k \cdot x} \int_{\mathbb{R}^{\mathbb{N}-\ell}} e^{i \xi \cdot y} e^{-t \hat{L}(k, \xi)} \hat{u}_{0}(k, \xi) d \xi .
$$

If we denote

$$
\bar{v}(t, y)=\frac{1}{(2 \pi)^{3-\ell}} \int_{\mathbb{R}^{\mathbb{N}-\ell}} e^{i \xi \cdot y} e^{-t \hat{L}(0, \xi)} \hat{u}_{0}(0, \xi) d \xi
$$

then it is easy to see that $\bar{v}(t, y)=(\bar{\phi}, \bar{m})(t, y)=\overline{\mathcal{U}}(t) \bar{u}_{0}$ is a solution to the following linear system

$$
\left\{\begin{array}{l}
\partial_{t} \bar{\phi}+\gamma \operatorname{div}^{\prime} \bar{m}^{\prime}=0 \\
\partial_{t} \bar{m}_{j}-\nu_{1} \Delta^{\prime} \bar{m}_{j}=0, j=1, \cdots, l \\
\partial_{t} \bar{m}^{\prime}-\nu_{1} \Delta^{\prime} \bar{m}^{\prime}-\nu_{2} \nabla^{\prime} \operatorname{div}^{\prime} \bar{m}^{\prime}+\gamma \nabla^{\prime} \bar{\phi}=0 \\
\bar{v}(0, y)=\left(\bar{\phi}_{0}, \bar{m}_{0}\right)(y)
\end{array}\right.
$$

where $\bar{m}^{\prime}=\left(\bar{m}_{\ell+1}, \cdots, \bar{m}_{3}\right)$.

In particular, if $\ell=3$, we can obtain from (2.6) that

$$
\left\{\begin{array}{l}
\partial_{t} \bar{\phi}=0 \\
\partial_{t} \bar{m}=0 \\
(\bar{\phi}, \bar{m})(0, y)=\left(\bar{\phi}_{0}, \bar{m}_{0}\right),
\end{array}\right.
$$

which implies $\bar{\phi}=\bar{\phi}_{0}$ and $\bar{m}=\bar{m}_{0}$. This, together with (2.5), yields $\left\|v-\bar{u}_{0}\right\|_{H^{s}} \leq C e^{-a_{0} t}\left\|u_{0}\right\|_{H^{s}}$ for $s \geq 0$.

Next we study the decay properties of $v$ in the cases of $\ell=1$ and $\ell=2$. Let $r_{0}$ be a positive constant defined in (2.5), and $\chi(z)$ be a function such that $\hat{\chi}(k, \xi)=\hat{\chi}(q)=1_{|q| \leq r_{0}}(k, \xi)$ with $|q|=\sqrt{k^{2}+|\xi|^{2}}$. As in [7-9], we set

$$
\mathcal{U}_{(0)}(t) u_{0}=\mathcal{F}^{-1}\left(e^{-t \hat{L}(k, \xi)} \hat{\chi}(q) \hat{u}_{0}\right), \mathcal{U}_{(\infty)}(t) u_{0}=\mathcal{F}^{-1}\left(e^{-t \hat{L}(k, \xi)}(1-\hat{\chi}(q)) \hat{u}_{0}\right),
$$




$$
\overline{\mathcal{U}}_{(0)}(t) u_{0}=\mathcal{F}^{-1}\left(e^{-t \hat{L}(0, \xi)} \hat{\chi}(0, \xi) \hat{u}_{0}\right), \overline{\mathcal{U}}_{(\infty)}(t) u_{0}=\mathcal{F}^{-1}\left(e^{-t \hat{L}(0, \xi)}(1-\hat{\chi}(0, \xi)) \hat{u}_{0}\right) .
$$

Then we have following results.

Lemma 2.1. For $\ell=1,2$ and $u_{0} \in H^{s}(\Omega) \cap L^{1}(\Omega)$ with $\Omega=\mathbb{T}^{\ell} \times \mathbb{R}^{3-\ell}, s \in \mathbb{N}$, then one has

(i) $\left\|\partial_{y}^{j} \mathcal{U}_{(0)}(t) u_{0}\right\|_{L^{2}(\Omega)} \leq C_{j}(1+t)^{-\frac{3-\ell}{4}-\frac{j}{2}}\left\|u_{0}\right\|_{L^{1}(\Omega)}, j \geq 0$.

(ii) $\left(\mathcal{U}_{(0)}(t) u_{0}\right)(t, z)=\bar{v}(t, y)$, and further $\partial_{x}^{j} \mathcal{U}_{(0)}(t) u_{0}=0$ for $j \geq 1$.

(iii) $\left\|\mathcal{U}_{(\infty)}(t) u_{0}\right\|_{H^{s}(\Omega)} \leq C e^{-a_{0} t}\left\|u_{0}\right\|_{H^{s}(\Omega)}$.

(iv) $\left\|\overline{\mathcal{U}}_{(\infty)}(t) \bar{u}_{0}\right\|_{H^{s}\left(\mathbb{R}^{3-\ell}\right)} \leq C e^{-a_{0} t}\left\|u_{0}\right\|_{H^{s}(\Omega)}$.

Where $a_{0}$ is a positive constant such that $\frac{a}{2} \leq a_{0} \leq a$ holds, and the constant $a$ is defined in (2.5).

Proof. (i) It follows from the Parseval's identity and a direct computation that

$$
\begin{aligned}
\left\|\partial_{y}^{j} \mathcal{U}_{(0)}(t) u_{0}\right\|_{L^{2}(\Omega)}^{2} & =\int_{|\xi| \leq r_{0}}|\xi|^{2 j}\left|e^{-t \hat{L}(0, \xi)} \hat{\chi}(0, \xi) \hat{u}_{0}(0, \xi)\right|^{2} d \xi \\
& \leq\left\|\hat{u}_{0}\right\|_{L^{\infty}(\Omega)}^{2} \int_{|\xi| \leq r_{0}}|\xi|^{2 j}\left|e^{-t \hat{L}(0, \xi)}\right|^{2} d \xi \\
& \leq C\left\|u_{0}\right\|_{L^{1}(\Omega)}^{2} \int_{|\xi| \leq r_{0}}|\xi|^{2 j} e^{-2 a_{0} t|\xi|^{2}} d \xi \\
& \leq|Z u| \leq\left\{\begin{array}{c}
C t^{-\frac{3-\ell}{2}-j}\left\|u_{0}\right\|_{L^{1}(\Omega)}^{2}, \\
C\left\|u_{0}\right\|_{L^{1}(\Omega)}^{2} .
\end{array}\right.
\end{aligned}
$$

(ii) follows the fact of $\mathcal{U}_{(0)}(t) u_{0}=\mathcal{U}_{(0)}(t) \bar{u}_{0}$ directly.

By (2.5), we have for $|k|^{2}+|\xi|^{2}>r_{0}$

$$
\left|e^{-t \hat{L}(0, \xi)}\right| \leq e^{-a t} .
$$

Therefore,

$$
\begin{aligned}
\left\|\partial_{x}^{j_{1}} \partial_{y}^{j_{2}} \mathcal{U}_{(\infty)}(t) u_{0}\right\|_{L^{2}(\Omega)} & =\left\|\mathcal{F}^{-1}\left(e^{-t \hat{L}(k, \xi)}(1-\hat{\chi}(p)) k^{j_{1}} \xi^{j_{2}} \hat{u}_{0}\right)\right\|_{L^{2}(\Omega)} \\
& \leq C e^{-a_{0} t}\left\|\partial_{x}^{j_{1}} \partial_{y}^{j_{2}} u_{0}\right\|_{L^{2}(\Omega)},
\end{aligned}
$$

which derives (iii) and (iv).

\section{§3. The global existence of the solution to the auxiliary problem (1.7)}

In this section, we shall establish the global existence of the classical solution $\eta(t, y)$ to (1.7) by the energy method. For notational convenience, we set $h_{1}=\left(\begin{array}{c}-\operatorname{div}^{\prime}\left(w^{\prime} \otimes w_{1}\right) \\ -\operatorname{div}^{\prime}\left(w^{\prime} \otimes w^{\prime}\right)-\alpha \nabla^{\prime}\left(\sigma^{2}\right)\end{array}\right)$. Then we have

Lemma 3.1. For small $\varepsilon>0, \eta_{0} \in H^{s}\left(\mathbb{R}^{2}\right)$ and $\left\|\eta_{0}\right\|_{H^{s}} \leq \varepsilon$, here $s \in \mathbb{N}$ and $s \geq 2$, then (1.7) admits a global solution $\eta(t, y)$ such that

$$
\eta \in C\left([0, \infty), H^{s}\left(\mathbb{R}^{2}\right)\right) \cap C^{1}\left([0, \infty), H^{s-2}\left(\mathbb{R}^{2}\right)\right) \text { and }\|\eta\|_{H^{s}} \leq C \varepsilon .
$$


Proof. Since the system in (1.7) is like the Navier-Stokes equation (1.1) and the local wellposedness of the initial data problem for the compressible Navier-Stokes system is well-known, we can derive that (1.7) is locally well-posed, and we omit the proof details here. In order to show the global well-posedness of (1.7), we only need to establish the related global energy estimates by the continuity argument.

Set $T^{j, k}=\partial_{t}^{j} \partial_{y}^{k}$. By $\int T^{j, k}(1.7) \cdot T^{j, k} \eta d y$ and a direct computation, we have

$$
\frac{1}{2} \frac{d}{d t}\left\|T^{j, k} \eta\right\|_{2}^{2}+\nu_{1}\left\|T^{j, k} \nabla^{\prime} w\right\|_{2}^{2}+\nu_{2}\left\|\operatorname{div}^{\prime} T^{j, k} w^{\prime}\right\|_{2}^{2}=\left(T^{j, k} h_{1}, T^{j, k} w\right) .
$$

In addition, it follows from $\int T^{j, k}(1.7)_{1} \times T^{j+1, k} \sigma d y$ and $\sum_{i=1}^{3} \int T^{j, k}(1.7)_{i+1} \times T^{j+1, k} w_{i} d y$ that

$$
\left\|T^{j+1, k} \sigma\right\|_{2}^{2}+\gamma\left(T^{j, k} \operatorname{div}^{\prime} w^{\prime}, T^{j+1, k} \sigma\right)=0
$$

and

$$
\begin{aligned}
& \left\|T^{j+1, k} w\right\|_{2}^{2}+\frac{1}{2} \frac{d}{d t}\left(\nu_{1}\left\|T^{j, k} \nabla^{\prime} w\right\|_{2}^{2}+\nu_{2}\left\|T^{j, k} \operatorname{div}^{\prime} w^{\prime}\right\|_{2}^{2}\right)+\gamma\left(T^{j, k} \nabla^{\prime} \sigma, T^{j+1, k} w^{\prime}\right) \\
& =\left(T^{j, k} h_{1}, T^{j+1, k} w\right) .
\end{aligned}
$$

Note that

$$
\left(T^{j, k} \nabla^{\prime} \sigma, T^{j+1, k} w^{\prime}\right)=-\frac{d}{d t}\left(T^{j, k} \sigma, T^{j, k} \operatorname{div}^{\prime} w^{\prime}\right)+\left(T^{j+1, k} \sigma, T^{j, k} \operatorname{div}^{\prime} w^{\prime}\right) .
$$

Together with (3.2)-(3.3) and Young's inequality, this yields

$$
\begin{gathered}
\frac{1}{2}\left\|T^{j+1, k} \sigma\right\|_{2}^{2}+\left\|T^{j+1, k} w\right\|_{2}^{2}+\frac{d}{d t}\left[\frac{\nu_{1}}{2}\left\|T^{j, k} \nabla^{\prime} w\right\|_{2}^{2}+\frac{\nu_{2}}{2}\left\|T^{j, k} \operatorname{div}^{\prime} w^{\prime}\right\|_{2}^{2}-\gamma\left(T^{j, k} \sigma, T^{j, k} \operatorname{div}^{\prime} w^{\prime}\right)\right] \\
\leq 2 \gamma^{2}\left\|T^{j, k} \operatorname{div}^{\prime} w^{\prime}\right\|_{2}^{2}+\left(T^{j, k} h_{1}, T^{j+1, k} w\right) .
\end{gathered}
$$

Computing $(3.2) \times \frac{2 \gamma^{2}}{\nu_{2}}+(3.4)$, we arrive at

$$
\begin{aligned}
& \frac{1}{2}\left\|T^{j+1, k} \sigma\right\|_{2}^{2}+\left\|T^{j+1, k} w\right\|_{2}^{2}+\frac{2 \nu_{1} \gamma^{2}}{\nu_{2}}\left\|T^{j, k+1} w\right\|_{2}^{2} \\
& \quad+\frac{d}{d t}\left(\frac{\nu_{1}}{2}\left\|T^{j, k} \nabla^{\prime} w\right\|_{2}^{2}+\frac{\nu_{2}}{2}\left\|T^{j, k} \operatorname{div}^{\prime} w^{\prime}\right\|_{2}^{2}-\gamma\left(T^{j, k} \sigma, T^{j, k} \operatorname{div}^{\prime} w^{\prime}\right)+\frac{\gamma^{2}}{\nu_{2}}\left\|T^{j, k} \eta\right\|_{2}^{2}\right) \\
& \leq \frac{2 \gamma^{2}}{\nu_{2}}\left(T^{j, k} h_{1}, T^{j, k} w\right)+\left(T^{j, k} h_{1}, T^{j+1, k} w\right) .
\end{aligned}
$$

Substituting the inequality $\gamma\left(T^{j, k} \sigma, T^{j, k} \operatorname{div}^{\prime} w^{\prime}\right) \leq \frac{\nu_{2}}{2}\left\|T^{j, k} \operatorname{div}^{\prime} w^{\prime}\right\|_{2}^{2}+\frac{\gamma^{2}}{2 \nu_{2}}\left\|T^{j, k} \sigma\right\|_{2}^{2}$ into (3.5), we have

$$
\left\|T^{j+1, k} \eta\right\|_{2}^{2}+\left\|T^{j, k+1} w\right\|_{2}^{2}+\frac{d}{d t}\left(\left\|T^{j, k+1} w\right\|_{2}^{2}+\left\|T^{j, k} \eta\right\|_{2}^{2}\right)
$$




$$
\leq C\left(T^{j, k} h_{1}, T^{j, k} w\right)+C\left(T^{j, k} h_{1}, T^{j+1, k} w\right)
$$

We only prove Lemma 3.1 for the case $s=2$ since the cases of $s \geq 3$ can be treated anagously. For $j=k=0$, and $j=0, k=1$, we have from (3.6) respectively

$$
\left\|\partial_{t} \eta\right\|_{2}^{2}+\left\|\nabla^{\prime} w\right\|_{2}^{2}+\frac{d}{d t}\left(\left\|\nabla^{\prime} w\right\|_{2}^{2}+\|\eta\|_{2}^{2}\right) \leq C\left(h_{1}, w\right)+C\left(h_{1}, \partial_{t} w\right)
$$

and

$$
\left\|\partial_{t} \partial_{y} \eta\right\|_{2}^{2}+\left\|\partial_{y}^{2} w\right\|_{2}^{2}+\frac{d}{d t}\left(\left\|\partial_{y}^{2} w\right\|_{2}^{2}+\left\|\partial_{y} \eta\right\|_{2}^{2}\right) \leq C\left(\partial_{y} h_{1}, \partial_{y} w\right)+C\left(\partial_{y} h_{1}, \partial_{t} \partial_{y} w\right)
$$

In addition, it follows from (3.1) with $j=0$ and $k=2$ that

$$
\frac{d}{d t}\left\|\partial_{y}^{2} \eta\right\|_{2}^{2}+\left\|\partial_{y}^{3} w\right\|_{2}^{2} \leq C\left(\partial_{y}^{2} h_{1}, \partial_{y}^{2} w\right)
$$

Adding (3.7)-(3.9), and using $\left(\partial_{y} h_{1}, \partial_{y} w\right)=-\left(h_{1}, \partial_{y}^{2} w\right)$ and $\left(\partial_{y}^{2} h_{1}, \partial_{y}^{2} w\right)=-\left(\partial_{y} h_{1}, \partial_{y}^{3} w\right)$, we get

$$
\begin{aligned}
& \left\|\partial_{t} \eta\right\|_{H^{1}}^{2}+\left\|\partial_{y} w\right\|_{H^{2}}^{2}+\frac{d}{d t}\|\eta\|_{H^{2}}^{2} \\
& \leq C\left(h_{1}, w\right)+C\left(h_{1}, \partial_{t} w\right)+C\left(h_{1}, \partial_{y}^{2} w\right)+C\left(\partial_{y} h_{1}, \partial_{y}^{3} w\right)+C\left(\partial_{y} h_{1}, \partial_{t} \partial_{y} w\right) .
\end{aligned}
$$

On the other hand, by $\nabla^{\prime}(1.7)_{1}+(1.7)_{3}$, we have

$$
\partial_{t} \nabla^{\prime} \sigma+\gamma \nabla^{\prime} \sigma=-\partial_{t} w^{\prime}+\nu_{1} \Delta^{\prime} w^{\prime}+\left(\nu_{2}-\gamma\right) \nabla^{\prime} \operatorname{div}^{\prime} w^{\prime}-\operatorname{div}^{\prime}\left(w^{\prime} \otimes w^{\prime}\right)-\alpha \nabla\left(\sigma^{2}\right) .
$$

Computing $\int(3.11) \cdot \nabla^{\prime} \sigma d y$ yields

$$
\begin{aligned}
& \gamma\left\|\nabla^{\prime} \sigma\right\|_{2}^{2}+\frac{d}{d t}\left\|\nabla^{\prime} \sigma\right\|_{2}^{2} \\
& \left.\leq\left(-\partial_{t} w^{\prime}+\nu_{1} \Delta^{\prime} w^{\prime}+\left(\nu_{2}-\gamma\right) \nabla^{\prime} \operatorname{div}^{\prime} w^{\prime}-\operatorname{div}^{\prime}\left(w^{\prime} \otimes w^{\prime}\right)-\alpha \nabla\left(\sigma^{2}\right), \nabla^{\prime} \sigma\right)\right) \\
& \left.\leq \frac{\gamma}{2}\left\|\nabla^{\prime} \sigma\right\|_{2}^{2}+C\left(\left\|\partial_{t} w^{\prime}\right\|_{2}^{2}+\left\|\partial_{y}^{2} w^{\prime}\right\|_{2}^{2}\right)+\left(-\operatorname{div}^{\prime}\left(w^{\prime} \otimes w^{\prime}\right)-\alpha \nabla\left(\sigma^{2}\right), \nabla^{\prime} \sigma\right)\right),
\end{aligned}
$$

which derives

$$
\begin{aligned}
& \frac{\gamma}{2}\left\|\nabla^{\prime} \sigma\right\|_{2}^{2}+\frac{d}{d t}\left\|\nabla^{\prime} \sigma\right\|_{2}^{2} \\
& \left.\leq C\left(\left\|\partial_{t} w^{\prime}\right\|_{2}^{2}+\left\|\partial_{y}^{2} w^{\prime}\right\|_{2}^{2}\right)+\left(-\operatorname{div}^{\prime}\left(w^{\prime} \otimes w^{\prime}\right)-\alpha \nabla^{\prime}\left(\sigma^{2}\right), \nabla^{\prime} \sigma\right)\right) .
\end{aligned}
$$

Computing $\int \partial_{y}(3.11) \cdot \partial_{y} \nabla^{\prime} \sigma d y$, we have

$$
\begin{aligned}
& \gamma\left\|\partial_{y} \nabla^{\prime} \sigma\right\|_{2}^{2}+\frac{d}{d t}\left\|\partial_{y} \nabla^{\prime} \sigma\right\|_{2}^{2} \\
& \left.\leq\left(-\partial_{y} \partial_{t} w^{\prime}+\nu_{1} \partial_{y} \Delta^{\prime} w^{\prime}+\left(\nu_{2}-\gamma\right) \partial_{y} \nabla^{\prime} \operatorname{div}^{\prime} w^{\prime}-\partial_{y} \operatorname{div}^{\prime}\left(w^{\prime} \otimes w^{\prime}\right)-\alpha \partial_{y} \nabla\left(\sigma^{2}\right), \partial_{y} \nabla^{\prime} \sigma\right)\right)
\end{aligned}
$$


10

$$
\left.\leq \frac{\gamma}{2}\left\|\partial_{y} \nabla^{\prime} \sigma\right\|_{2}^{2}+C\left(\left\|\partial_{y} \partial_{t} w^{\prime}\right\|_{2}^{2}+\left\|\partial_{y}^{3} w^{\prime}\right\|_{2}^{2}\right)+\left(-\partial_{y} \operatorname{div}^{\prime}\left(w^{\prime} \otimes w^{\prime}\right)-\alpha \partial_{y} \nabla^{\prime}\left(\sigma^{2}\right), \partial_{y} \nabla^{\prime} \sigma\right)\right),
$$

which concludes

$\left.\frac{\gamma}{2}\left\|\partial_{y}^{2} \sigma\right\|_{2}^{2}+\frac{d}{d t}\left\|\partial_{y}^{2} \sigma\right\|_{2}^{2} \leq C\left(\left\|\partial_{y} \partial_{t} w^{\prime}\right\|_{2}^{2}+\left\|\partial_{y}^{3} w^{\prime}\right\|_{2}^{2}\right)+\left(-\partial_{y} \operatorname{div}^{\prime}\left(w^{\prime} \otimes w^{\prime}\right)-\alpha \partial_{y} \nabla^{\prime}\left(\sigma^{2}\right), \partial_{y} \nabla^{\prime} \sigma\right)\right)$.

Combining (3.10) with (3.12)-(3.13) yields

$$
\begin{aligned}
& \left\|\partial_{t} \eta\right\|_{H^{1}}^{2}+\left\|\partial_{y} w\right\|_{H^{2}}^{2}+\left\|\partial_{y} \sigma\right\|_{H^{1}}^{2}+\frac{d}{d t}\|\eta\|_{H^{2}}^{2} \\
& \leq C\left(h_{1}, w\right)+\left(h_{1}, \partial_{t} w\right)+C\left(h_{1}, \partial_{y}^{2} w\right)+C\left(\partial_{y} h_{1}, \partial_{y}^{3} w\right)+\left(\partial_{y} h_{1}, \partial_{t} \partial_{y} w\right) \\
& \quad+\left(-\operatorname{div}^{\prime}\left(w^{\prime} \otimes w^{\prime}\right)-\alpha \nabla^{\prime}\left(\sigma^{2}\right), \nabla^{\prime} \sigma\right)+\left(-\partial_{y} \operatorname{div}^{\prime}\left(w^{\prime} \otimes w^{\prime}\right)-\alpha \partial_{y} \nabla^{\prime}\left(\sigma^{2}\right), \partial_{y} \nabla^{\prime} \sigma\right) .
\end{aligned}
$$

Define

$$
N(t)=\|\eta\|_{H^{2}}^{2}+\int_{0}^{t}\left\{\left\|\partial_{t} \eta\right\|_{H^{1}}^{2}+\left\|\partial_{y} w\right\|_{H^{2}}^{2}+\left\|\partial_{y} \sigma\right\|_{H^{1}}^{2}\right\} d \tau
$$

Integrating (3.14) with respect to the variable $\tau$ over $(0, t)$, one has

$$
\begin{aligned}
N(t) & \leq\left\|\eta_{0}\right\|_{H^{2}}^{2}+C \int_{0}^{t}\left\{\left(h_{1}, w\right)+\left(h_{1}, \partial_{t} w\right)+\left(h_{1}, \partial_{y}^{2} w\right)+\left(\partial_{y} h_{1}, \partial_{y}^{3} w\right)+\left(\partial_{y} h_{1}, \partial_{t} \partial_{y} w\right)\right. \\
& \left.+\left(-\operatorname{div}^{\prime}\left(w^{\prime} \otimes w^{\prime}\right)-\alpha \nabla^{\prime}\left(\sigma^{2}\right), \nabla^{\prime} \sigma\right)+\left(-\partial_{y} \operatorname{div}^{\prime}\left(w^{\prime} \otimes w^{\prime}\right)-\alpha \partial_{y} \nabla^{\prime}\left(\sigma^{2}\right), \partial_{y} \nabla^{\prime} \sigma\right)\right\} d \tau .
\end{aligned}
$$

Next we treat the terms in the right hand side of (3.15) separately. For the term $\int_{0}^{t}\left(h_{1}, w\right) d \tau$, by using $(1.7)_{1}$, we see that

$$
\left(h_{1}, w\right)=\left(-\left(\operatorname{div}^{\prime}\left(w^{\prime} \otimes w_{1}\right), w_{1}\right)+\left(-\operatorname{div}^{\prime}\left(w^{\prime} \otimes w^{\prime}\right)-\alpha \nabla^{\prime}\left(\sigma^{2}\right), w^{\prime}\right) .\right.
$$

Noting that

$$
\begin{aligned}
\left(-\left(\operatorname{div}^{\prime}\left(w^{\prime} \otimes w_{1}\right), w_{1}\right)\right. & =\int w_{1} \cdot\left(w^{\prime} \cdot \nabla^{\prime}\right) w_{1} d y=\frac{1}{2} \int\left(w^{\prime} \cdot \nabla^{\prime}\right)\left|w_{1}\right|^{2} d y \\
& =-\frac{1}{2} \int\left|w_{1}\right|^{2} \operatorname{div}^{\prime} w^{\prime} d y=\frac{1}{2 \gamma} \int w_{1}^{2} \partial_{\tau} \sigma d y
\end{aligned}
$$

and

$$
\begin{aligned}
\left(-\operatorname{div}^{\prime}\left(w^{\prime} \otimes w^{\prime}\right)-\alpha \nabla^{\prime}\left(\sigma^{2}\right), w^{\prime}\right) & =-\frac{1}{2} \int\left(\operatorname{div}^{\prime} w^{\prime}\right)\left|w^{\prime}\right|^{2} d y+\int \alpha \sigma^{2} \operatorname{div}^{\prime} w^{\prime} d y \\
& =\frac{1}{2 \gamma} \int \partial_{\tau} \sigma\left|w^{\prime}\right|^{2} d y-\frac{1}{\gamma} \int \alpha \sigma^{2} \partial_{t} \sigma d y,
\end{aligned}
$$

then we have

$$
\int_{0}^{t}\left(h_{1}, w\right) d \tau=J_{1}+J_{2}
$$


where

$$
\begin{aligned}
& J_{1}=-\frac{\alpha}{\gamma} \int_{0}^{t} \int \partial_{\tau} \sigma \sigma^{2} d y d \tau \\
& J_{2}=\frac{1}{2 \gamma} \int_{0}^{t} \int \partial_{\tau} \sigma|w|^{2} d y d \tau
\end{aligned}
$$

It follows from a direct computation that

$$
\begin{aligned}
J_{1} & \leq C\left(\int \sigma^{3}(t, y) d y-\int \sigma^{3}(0, y) d y\right) \\
& \leq C\left(\|\sigma\|_{3}^{3}+\left\|\sigma_{0}\right\|_{3}^{3}\right) \leq C\left\|\sigma_{0}\right\|_{H^{2}}^{3}+C N^{3 / 2}(t)
\end{aligned}
$$

and

$$
J_{2}=\frac{1}{2 \gamma} \int_{0}^{t} \int \partial_{\tau}\left(\sigma|w|^{2}\right) d y d \tau-\frac{1}{\gamma} \int_{0}^{t} \int \sigma w \partial_{\tau} w d y d \tau
$$

For the first term in the right hand side of (3.18), we have

$$
\frac{1}{2 \gamma} \int_{0}^{t} \int \partial_{\tau}\left(\sigma|w|^{2}\right) d y d \tau \leq C \int \sigma_{0}\left|w_{0}\right|^{2} d y+C \int \sigma|w|^{2} d y \leq C\left\|\eta_{0}\right\|_{H^{2}}^{3}+C N^{3 / 2}(t)
$$

For the second term in the right hand side of (3.18), we have by $(1.7)_{2,3,4}$

$$
\begin{aligned}
& \frac{1}{\gamma} \int_{0}^{t} \int \sigma w \partial_{\tau} w d y d \tau \\
& \leq C \int_{0}^{t} \int \sigma\left\{\nu_{1} \Delta^{\prime} w \cdot w+\nu_{2} \nabla^{\prime} \operatorname{div}^{\prime} w^{\prime} \cdot w^{\prime}-\operatorname{div}^{\prime}\left(w^{\prime} \otimes w^{\prime}\right) w^{\prime}-\alpha \nabla^{\prime}\left(\sigma^{2}\right) w^{\prime}\right. \\
& \left.\quad-\operatorname{div}^{\prime}\left(w^{\prime} \otimes w_{1}\right) w_{1}-\gamma \nabla^{\prime} \sigma \cdot w^{\prime}\right\} d y d \tau .
\end{aligned}
$$

It is noted that

$$
\nu_{1} \int_{0}^{t} \int \sigma \Delta^{\prime} w \cdot w d y d \tau=-\nu_{1} \int_{0}^{t} \int \nabla^{\prime}(\sigma w) \nabla^{\prime} w d y d \tau \leq C \int_{0}^{t}\|\eta\|_{L^{\infty}}\|\nabla \eta\|_{2}^{2} d \tau \leq C N^{\frac{3}{2}}(t) .
$$

Similarly,

$$
\begin{gathered}
\int_{0}^{t} \int \nu_{2} \sigma \nabla^{\prime} \operatorname{div}^{\prime} w^{\prime} \cdot w^{\prime} d y d \tau \leq C N^{\frac{3}{2}}(t), \\
\int_{0}^{t} \int \sigma d i v^{\prime}\left(w^{\prime} \otimes w^{\prime}\right) w^{\prime} d y d \tau \leq C \int_{0}^{t}\|\nabla \eta\|_{2}\|\eta\|_{6}^{3} d \tau \leq C \int_{0}^{t}\|\nabla \eta\|_{2}\|\eta\|_{2}^{2}\left\|\nabla^{\prime} \eta\right\|_{2} d \tau \leq C N^{2}(t) .
\end{gathered}
$$

Then substituting the above three estimates into (3.20) and subsequently combining (3.17)(3.19) yield

$$
\int_{0}^{t}\left(h_{1}, w\right) d \tau \leq\left\|\eta_{0}\right\|_{H^{2}}^{2}+C N^{\frac{3}{2}}(t)
$$


12

For the other left terms in the right hand side of (3.15), we have

$$
\begin{aligned}
\int_{0}^{t}\left(h_{1}, \partial_{\tau} w\right) d \tau & \leq \int_{0}^{t} \int\left|\partial_{y} \eta\|\eta\| \partial_{\tau} w\right| d y d \tau \\
& \leq C\|\eta\|_{L^{\infty}} \int_{0}^{t}\left(\left\|\partial_{y} \eta\right\|_{2}^{2}+\left\|\partial_{t} w\right\|_{2}^{2}\right) d y d \tau \\
& \leq C N^{3 / 2}(t)
\end{aligned}
$$

and

$$
\begin{aligned}
\int_{0}^{t}\left(h_{1}, \partial_{y}^{2} w\right) d \tau & \leq \int_{0}^{t} \int\left|\partial_{y} \eta\|\eta\| \partial_{y}^{2} w\right| d y d \tau \\
& \leq C\|\eta\|_{L^{\infty}} \int_{0}^{t}\left(\left\|\partial_{y} \eta\right\|_{2}^{2}+\left\|\partial_{y}^{2} w\right\|_{2}^{2}\right) d y d \tau \\
& \leq C N^{3 / 2}(t)
\end{aligned}
$$

and

$$
\begin{aligned}
& \int_{0}^{t}\left(h_{1}, \partial_{y} \sigma\right) d \tau \leq C N^{3 / 2}(t) \\
& \int_{0}^{t}\left(\partial_{y} h_{1}, \partial_{t} \partial_{y} w\right) d \tau \leq \int_{0}^{t} \int\left|\partial_{t} \partial_{y} w\right|\left(|\eta|\left|\partial_{y} \eta\right|^{2}+|\eta|^{2}\left|\partial^{2} \eta\right|\right) d y d \tau \\
& \leq C\|\eta\|_{L^{\infty}} \int_{0}^{t}\left\|\partial_{t} \partial_{y} w\right\|\left\|\partial_{y} \eta\right\|_{L^{4}}^{2} d \tau+C\|\eta\|_{L^{\infty}}^{2} \int_{0}^{t}\left\|\partial_{t} \partial_{y} w\right\|\left\|\partial_{y}^{2} \eta\right\| d \tau \\
& \leq C N^{3 / 2}(t) \\
& \int_{0}^{t}\left(\partial_{y} h_{1}, \partial_{y}^{3} w\right) d \tau \leq C \int_{0}^{t} \int\left(\left|\partial_{y}^{2} \eta\right||\eta|+\left|\partial_{y} \eta\right|^{2}\right)\left|\partial_{y}^{3} w\right| d y d \tau \\
& \leq C\|\eta\|_{L^{\infty}} \int_{0}^{t} \int\left|\partial_{y}^{2} \eta\left\|\partial_{y}^{3} w \mid d y d \tau+C \int_{0}^{t}\right\| \partial_{y}^{3} w\left\|_{2}\right\| \partial_{y} \eta \|_{4}^{2} d \tau\right. \\
& \leq C\|\eta\|_{L^{\infty}} \int_{0}^{t} \int\left|\partial_{y}^{2} \eta\left\|\partial_{y}^{3} w \mid d y d \tau+C \int_{0}^{t}\right\| \partial_{y}^{3} w\left\|_{2}\right\| \partial_{y} \eta\left\|_{2}\right\| \partial_{y}^{2} \eta \|_{2} d \tau\right. \\
& \leq C N^{3 / 2}(t) \\
& \int_{0}^{t}\left(\partial_{y} h_{1}, \partial_{y}^{2} \sigma\right) d \tau \leq C \int_{0}^{t} \int\left(\left|\partial_{y}^{2} \eta\right||\eta|+\left|\partial_{y} \eta\right|^{2}\right)\left|\partial_{y}^{2} \sigma\right| d y d \tau \\
& \leq C\|\eta\|_{L^{\infty}} \int_{0}^{t} \int\left|\partial_{y}^{2} \eta\left\|\partial_{y}^{2} \sigma \mid d y d \tau+C \int_{0}^{t}\right\| \partial_{y}^{2} \sigma\left\|_{2}\right\| \partial_{y} \eta \|_{4}^{2} d \tau\right. \\
& \leq C\|\eta\|_{L^{\infty}} \int_{0}^{t} \int\left|\partial_{y}^{2} \eta\left\|\partial_{y}^{2} \sigma \mid d y d \tau+C \int_{0}^{t}\right\| \partial_{y}^{2} \sigma\left\|_{2}\right\| \partial_{y} \eta\left\|_{2}\right\| \partial_{y}^{2} \eta \|_{2} d \tau\right. \\
& \leq C N^{3 / 2}(t) \text {. }
\end{aligned}
$$

Therefore, substituting (3.21)-(3.27) into (3.15) yields

$$
N(t) \leq C \varepsilon^{2}+C N^{3 / 2}(t)
$$


which implies $N(t) \leq C \varepsilon^{2}$ holds for all $t \in[0,+\infty)$. By the local existence of the solution to (1.7) and the continuity argument, we know that Lemma 3.1 holds for $s=2$. Analogously, Lemma 3.1 also holds for $s \geq 3$.

Next we establish the decay property of the solution to (1.7). For notational convenience, we write (1.7) as

$$
\partial_{t} \eta+A \eta=B(\eta)
$$

where

$$
A \eta=\left(\begin{array}{c}
\gamma \operatorname{div}^{\prime} w^{\prime} \\
-\nu_{1} \Delta^{\prime} w_{1} \\
-\nu_{1} \Delta^{\prime} w^{\prime}-\nu_{2} \nabla^{\prime} \operatorname{div}^{\prime} w^{\prime}+\gamma \nabla^{\prime} \sigma
\end{array}\right)
$$

and

$$
B(\eta)=\left(\begin{array}{c}
0 \\
-\operatorname{div}^{\prime}\left(w^{\prime} \otimes w_{1}\right) \\
-\operatorname{div}^{\prime}\left(w^{\prime} \otimes w^{\prime}\right)-\alpha \nabla^{\prime}\left(\sigma^{2}\right)
\end{array}\right) .
$$

Noting that $\hat{A \eta}=\hat{L}(0, \xi) \hat{\eta}$, where $\hat{L}(0, \xi)$ has been defined in (2.2), then by Duhamel's principle, we have from (3.28)

$$
\eta(t, y)=e^{-t A} \eta_{0}+\int_{0}^{t} e^{-(t-\tau) A} B(\eta)(\tau) d \tau .
$$

Lemma 3.2. Set $M_{0}(t)=\sup _{0 \leq \tau \leq t}\left\{(1+\tau)^{\frac{1}{2}}\|\eta\|_{2}+(1+\tau)\left\|\partial_{y} \eta\right\|_{2}\right\}$. If $\eta_{0}(y)=\left(\sigma_{0}, w_{0}\right)(y) \in$ $H^{4}\left(\mathbb{R}^{2}\right) \cap L^{1}\left(\mathbb{R}^{2}\right)$ and $\left\|\eta_{0}\right\|_{H^{4} \cap L^{1}} \leq \varepsilon$, then we have for small $\varepsilon>0$

(i) $\left\|\partial_{y}^{j} e^{-t A} \eta_{0}\right\|_{2} \leq C_{j}\left((1+t)^{-\frac{1}{2}-\frac{j}{2}}\left\|\eta_{0}\right\|_{L^{1}}+e^{-a_{0} t}\left\|\eta_{0}\right\|_{H^{j}}\right), \quad j=0,1, \cdots, 4$.

(ii) $M_{0}(t) \leq C\left(\left\|\eta_{0}\right\|_{L^{1}}+\left\|\eta_{0}\right\|_{H^{4}}\right)+C\left(M_{0}^{2}(t)+\varepsilon^{\frac{1}{4}} M_{0}^{\frac{17}{12}}(t)\right)$.

Where $a_{0}$ is a positive constant such that $\frac{a}{2} \leq a_{0} \leq a$ holds, and the constant $a>0$ is defined in (2.5).

Proof. (i) Its proof is completely similar to that for Lemma 2.1 (i) and (iii), we omit it here. (ii) From (3.29) we see that

$$
\left\|\partial_{y}^{j} \eta(t, y)\right\|_{2}=\left\|\partial_{y}^{j} e^{-t A} \eta_{0}\right\|_{2}+\int_{0}^{t}\left\|e^{-(t-\tau) A} \partial_{y}^{j} B(\eta)\right\|_{2}(\tau) d \tau .
$$

Since the first term $\left\|\partial_{y}^{j} e^{-t A} \eta_{0}\right\|_{2}$ in the right hand side of (3.30) has been treated in (i), we only need to treat the second term. For the nonlinear term $B(\eta)$, we observe that

$$
B(\eta)=\operatorname{div}^{\prime} b(\eta),
$$

where

$$
b(\eta)=\left(\begin{array}{c}
0 \\
-w^{\prime} \otimes w_{1} \\
-w^{\prime} \otimes w^{\prime}-\alpha \sigma^{2} I_{2}
\end{array}\right) .
$$


It follows from (i) that

$$
\left\|e^{-(t-\tau) A} B(\eta)\right\|_{2} \leq C\left((1+t-\tau)^{-\frac{1}{2}}\|B(\eta)\|_{L^{1}}+e^{-a_{0}(t-\tau)}\|B(\eta)\|_{L^{2}}\right)
$$

and

$$
\left\|e^{-(t-\tau) A} B(\eta)\right\|_{2}=\left\|\operatorname{div}^{\prime} e^{-(t-\tau) A} b(\eta)\right\|_{2} \leq C\left((1+t-\tau)^{-1}\|b(\eta)\|_{L^{1}}+e^{-a_{0}(t-\tau)}\left\|\partial_{y} b(\eta)\right\|_{L^{2}}\right) .
$$

In addition, we have

$$
\int_{0}^{t}\left\|e^{-(t-\tau) A} B(\eta)\right\|_{2}(\tau) d \tau=\int_{0}^{t / 2}\left\|\partial_{y} e^{-(t-\tau) A} b(\eta)\right\|_{2}(\tau) d \tau+\int_{t / 2}^{t}\left\|e^{-(t-\tau) A} B(\eta)\right\|_{2}(\tau) d \tau
$$

For the first term in the right hand side of (3.33), one has by (3.32)

$$
\begin{aligned}
& \int_{0}^{t / 2}\left\|\partial_{y} e^{-(t-\tau) A} b(\eta)\right\|_{2}(\tau) d \tau \\
& \leq C \int_{0}^{t / 2}\left((1+t-\tau)^{-1}\|b(\eta)\|_{L^{1}}+e^{-a_{0}(t-\tau)}\left\|\partial_{y} b(\eta)\right\|_{L^{2}}\right) d \tau \\
& \leq C \int_{0}^{t / 2}\left((1+t-\tau)^{-1}\|\eta\|_{2}^{2}+e^{-a_{0}(t-\tau)}\|\eta\|_{L^{4}}\left\|\partial_{y} \eta\right\|_{L^{4}}\right) d \tau \\
& \leq C \int_{0}^{t / 2}\left((1+t-\tau)^{-1}(1+\tau)^{-1} M_{0}^{2}(\tau)+\varepsilon^{\frac{1}{4}} e^{-a_{0}(t-\tau)}(1+\tau)^{-\frac{13}{8}} M_{0}^{\frac{7}{4}}(\tau)\right) d \tau \\
& \leq C(1+t)^{-1} \ln (1+t) M_{0}^{2}(t)+\varepsilon^{\frac{1}{4}} e^{-\frac{a_{0}}{4} t} M_{0}^{\frac{7}{4}}(t)
\end{aligned}
$$

For the second term of (3.33), then it follows from (3.31) that

$$
\begin{aligned}
& \int_{t / 2}^{t}\left\|e^{-(t-\tau) A} B(\eta)\right\|_{2}(\tau) d \tau \\
& \leq C \int_{t / 2}^{t}\left((1+t-\tau)^{-\frac{1}{2}}\|B(\eta)\|_{L^{1}}+e^{-a_{0}(t-\tau)}\|B(\eta)\|_{L^{2}}\right) d \tau \\
& \leq C \int_{t / 2}^{t}\left((1+t-\tau)^{-\frac{1}{2}}\left\|\partial_{y} \eta\right\|_{2}\|\| \eta\left\|_{2}+e^{-a_{0}(t-\tau)}\right\| \eta\left\|_{L^{4}}\right\| \partial_{y} \eta \|_{L^{4}}\right) d \tau \\
& \leq C \int_{t / 2}^{t}\left((1+t-\tau)^{-\frac{1}{2}}(1+\tau)^{-\frac{3}{2}} M_{0}^{2}(\tau)+\varepsilon^{\frac{1}{4}} e^{-a_{0}(t-\tau)}(1+\tau)^{-\frac{13}{8}} M_{0}^{\frac{7}{4}}(\tau)\right) d \tau \\
& \leq C(1+t)^{-1} M_{0}^{2}(t)+C \varepsilon^{\frac{1}{4}}(1+t)^{-\frac{13}{8}} M_{0}^{\frac{7}{4}}(t)
\end{aligned}
$$

Therefore, combining (3.34) with (3.35), we can obtain the estimate (3.30) with $j=0$.

In the case of $j=1$, we have

$$
\int_{0}^{t / 2}\left\|\partial_{y}^{2} e^{-(t-\tau) A} b(\eta)\right\|_{2}(\tau) d \tau
$$




$$
\begin{aligned}
& \leq C \int_{0}^{t / 2}\left((1+t-\tau)^{-\frac{3}{2}}\|b(\eta)\|_{L^{1}}+e^{-a_{0}(t-\tau)}\left\|\partial_{y}^{2} b(\eta)\right\|_{L^{2}}\right) d \tau \\
& \leq C \int_{0}^{t / 2}\left((1+t-\tau)^{-\frac{3}{2}}\|\eta\|_{2}^{2}+e^{-a_{0}(t-\tau)}\left(\left\|\partial_{y} \eta \cdot \partial_{y} \eta\right\|_{L^{2}}+\left\|\eta \cdot \partial_{y}^{2} \eta\right\|_{L^{2}}\right)\right) d \tau \\
& \leq C \int_{0}^{t / 2}\left((1+t-\tau)^{-\frac{3}{2}}(1+\tau)^{-1} M_{0}^{2}(\tau)+e^{-a_{0}(t-\tau)}\left(\left\|\partial_{y} \eta\right\|_{L^{4}}^{2}+\|\eta\|_{L^{4}}\left\|\partial_{y}^{2} \eta\right\|_{L^{4}}\right)\right) d \tau \\
& \leq C \int_{0}^{t / 2}\left((1+t-\tau)^{-\frac{3}{2}}(1+\tau)^{-1} M_{0}^{2}(\tau)+e^{-a_{0}(t-\tau)}\left(\varepsilon^{\frac{1}{2}}(1+\tau)^{-\frac{3}{2}} M_{0}^{\frac{3}{2}}(\tau)+\varepsilon^{\frac{7}{12}}(1+\tau)^{-\frac{3}{2}} M_{0}^{\frac{17}{12}}(\tau)\right)\right) d \tau \\
& \leq C(1+t)^{-\frac{3}{2}} \ln (1+t) M_{0}^{2}(t)+\varepsilon^{\frac{1}{2}} e^{-\frac{a_{0}}{4} t} M_{0}^{\frac{17}{12}}(t)
\end{aligned}
$$

and

$$
\begin{aligned}
& \int_{t / 2}^{t}\left\|\partial_{y} e^{-(t-\tau) A} B(\eta)\right\|_{2}(\tau) d \tau \\
\leq & C \int_{t / 2}^{t}\left((1+t-\tau)^{-1}\|B(\eta)\|_{L^{1}}+e^{-a_{0}(t-\tau)}\left\|\partial_{y} B(\eta)\right\|_{L^{2}}\right) d \tau \\
\leq & C \int_{t / 2}^{t}\left((1+t-\tau)^{-1}\left\|\partial_{y} \eta\right\|_{2}\|\| \eta \|_{2}+e^{-a_{0}(t-\tau)}\left(\left\|\partial_{y} \eta \cdot \partial_{y} \eta\right\|_{L^{2}}+\left\|\eta \cdot \partial_{y}^{2} \eta\right\|_{L^{2}}\right)\right) d \tau \\
\leq & C \int_{t / 2}^{t}\left((1+t-\tau)^{-1}(1+\tau)^{-\frac{3}{2}} M_{0}^{2}(\tau)+e^{-a_{0}(t-\tau)}\left(\varepsilon^{\frac{1}{2}}(1+\tau)^{-\frac{3}{2}} M_{0}^{\frac{3}{2}}(\tau)+\varepsilon^{\frac{7}{12}}(1+\tau)^{-\frac{3}{2}} M_{0}^{\frac{17}{12}}(\tau)\right)\right) d \tau \\
\leq & C\left((1+t)^{-\frac{3}{2}} \ln (1+t) M_{0}^{2}(t)+\varepsilon^{\frac{1}{2}}(1+\tau)^{-\frac{3}{2}} M_{0}^{\frac{3}{2}}(t)+\varepsilon^{\frac{7}{12}}(1+\tau)^{-\frac{3}{2}} M_{0}^{\frac{17}{12}}(t)\right) .
\end{aligned}
$$

Combining (3.34)-(3.37) yields for $j=0,1$,

$$
\left\|\partial_{y}^{j} \eta\right\| \leq C(1+t)^{-\frac{1}{2}-\frac{j}{2}}\left\|\eta_{0}\right\|_{H^{4} \cap L^{1}}+C(1+t)^{-\frac{1}{2}-\frac{j}{2}}\left(M_{0}^{2}(t)+\varepsilon^{\frac{1}{4}} M_{0}^{\frac{17}{12}}(t)\right) .
$$

Consequently, we complete the proof of (ii).

Remark 3.1. By analogous arguments as in Lemma 3.2, for the problem (1.11), if $\eta_{0}(y)=$ $\left(\sigma_{0}, w_{0}\right)(y) \in H^{4}(\mathbb{R}) \cap L^{1}(\mathbb{R})$ and $\left\|\eta_{0}\right\|_{H^{4} \cap L^{1}} \leq \varepsilon$, then we have for small $\varepsilon>0$

$$
\tilde{M}_{0}(t) \leq C\left(\left\|\eta_{0}\right\|_{L^{1}}+\left\|\eta_{0}\right\|_{H^{4}}\right)+C\left(\tilde{M}_{0}^{2}(t)+\varepsilon^{\frac{1}{4}} \tilde{M}_{0}^{\frac{17}{12}}(t)\right),
$$

where $\tilde{M}_{0}(t)=\sup _{0 \leq \tau \leq t}\left\{(1+\tau)^{\frac{1}{4}}\|\eta\|_{2}+(1+\tau)^{\frac{3}{4}}\left\|\partial_{y} \eta\right\|_{2}\right\}$.

\section{$\S 4$. Some decay properties of the solution $u(t, z)$ to (1.3) for $z \in \mathbb{T} \times \mathbb{R}^{2}$}

In this section, we will study the large time behavior of the solution to (1.3) for $z \in \mathbb{T} \times \mathbb{R}^{2}$ as $t$ tends to infinity. As an ingredient of the proof, we require to cite the following GagliardoNirenberg-Sobolev inequalities (see [1] and so on) which will be used repeatedly.

Lemma 4.1.(Gagliardo-Nirenberg-Sobolev) (i) Let $2 \leq p \leq \infty$, and let $j$ and $k$ be the integers satisfying

$$
0 \leq j<k, k>j+n\left(\frac{1}{2}-\frac{1}{p}\right) .
$$


Then for $f \in H^{k}\left(\mathbb{R}^{n}\right)$, there exists a constant $C>0$ independent of $f$ such that

$$
\left\|\partial_{x}^{j} f\right\|_{L^{p}\left(\mathbb{R}^{n}\right)} \leq C\|f\|_{L^{2}\left(\mathbb{R}^{n}\right)}^{1-a}\left\|\partial_{x}^{k} f\right\|_{L^{2}\left(\mathbb{R}^{n}\right)}^{a},
$$

where $a=\frac{1}{k}\left(j+\frac{n}{2}-\frac{n}{p}\right)$.

(ii) If $f \in H^{1}(\mathbb{R})$, then

$$
\|f\|_{L^{\infty}} \leq C\|f\|_{L^{2}}^{\frac{1}{2}}\left\|\partial_{x} f\right\|_{L^{2}}^{\frac{1}{2}}
$$

Define

$$
\Pi[g](y)=\bar{g}(y)=\frac{1}{2 \pi} \int_{\mathbb{T}} g(x, y) d x .
$$

By Duhamel's principle, one can get the follow expressions of the solutions to (1.3)

$$
u(t, z)=\mathcal{U}(t) u_{0}+\int_{0}^{t} \mathcal{U}(t-\tau) f(\tau, z) d \tau
$$

and

$$
\bar{u}(t, y)=\overline{\mathcal{U}}(t) \bar{u}_{0}+\int_{0}^{t} \overline{\mathcal{U}}(t-\tau) \bar{f}(\tau, y) d \tau,
$$

where $f(t, z)=\left(\begin{array}{c}0 \\ G(\phi, m)\end{array}\right)$ and $\bar{f}(t, y)=\Pi[f](t, y)$.

We note that $G(\phi, m)$ in $(1.3)$ can be written as

$$
G=\operatorname{div} \mathcal{G}(\phi, m)+\nabla g(\phi, m)
$$

where

$$
\begin{aligned}
\mathcal{G}(\phi, m) & =-\frac{\gamma}{\phi+\gamma} m \otimes m-\nu_{1} \nabla \otimes\left(\frac{\phi}{\phi+\gamma} m\right), \\
g(\phi, m) & =-\nu_{2} \operatorname{div}\left(\frac{\phi}{\phi+\gamma} m\right)-F(\phi)
\end{aligned}
$$

and $F(\phi)=\frac{\phi^{2}}{\gamma^{2}} \int_{0}^{1}(1-\theta)^{2} p^{\prime \prime}\left(1+\frac{\theta \phi}{\gamma}\right) d \theta$

Due to $\Pi[\operatorname{div} v]=\Pi\left[\operatorname{div}^{\prime} v^{\prime}\right]$ and $\Pi[\nabla f]=\left(0, \nabla^{\prime} \bar{f}\right)^{T}, \bar{u}(t, y)=(\bar{\phi}, \bar{m})(t, y)$ satisfies the nonlinear system

$$
\left\{\begin{array}{l}
\partial_{t} \bar{\phi}+\gamma \operatorname{div}^{\prime} \bar{m}^{\prime}=0 \\
\partial_{t} \bar{m}_{1}-\nu_{1} \Delta^{\prime} \bar{m}_{1}=-\Pi\left[\operatorname{div}^{\prime}\left(\frac{\gamma}{\phi+\gamma} m_{1} m^{\prime}\right)-\nu_{1} \Delta^{\prime}\left(\frac{\phi}{\phi+\gamma} m_{1}\right)\right] \\
\partial_{t} \bar{m}^{\prime}-\nu_{1} \Delta^{\prime} \bar{m}^{\prime}-\nu_{2} \nabla^{\prime} \operatorname{div}^{\prime} \bar{m}^{\prime}+\gamma \nabla^{\prime} \bar{\phi}=\Pi\left[G^{\prime}\left(\phi, m^{\prime}\right)\right] \\
\bar{u}(0, y)=\left(\bar{\phi}_{0}, \bar{m}_{0}\right)(y)
\end{array}\right.
$$


where

$$
G^{\prime}\left(\phi, m^{\prime}\right)=-\operatorname{div}^{\prime}\left(\frac{\gamma}{\phi+\gamma} m^{\prime} \otimes m^{\prime}\right)-\nu_{1} \Delta^{\prime}\left(\frac{\phi}{\phi+\gamma} m^{\prime}\right)-\nu_{2} \nabla^{\prime} \operatorname{div}^{\prime}\left(\frac{\phi}{\phi+\gamma} m^{\prime}\right)-\nabla^{\prime} F(\phi) .
$$

Let $\tilde{u}=u-\bar{u}$ and

$$
M(t)=M[u](t) \equiv \sup _{0 \leq \tau \leq t}\left\{(1+\tau)^{\frac{1}{2}}\|u(\tau, z)\|_{L^{2}(\Omega)}+(1+\tau)\|\nabla u(\tau, z)\|_{L^{2}(\Omega)}\right\}
$$

Noting that $\bar{u}(t, y)$ is the mean value of $u(t, z)$ with respect to the periodic variable $x$, then one has $M[\bar{u}](t) \leq C M(t)$ and further $M[\tilde{u}](t) \leq C M(t)$.

We now establish the following decay estimates on the solution $u$ to (1.3).

Lemma 4.2. Assume $u \in C\left([0,+\infty), H^{4}\left(\mathbb{T} \times \mathbb{R}^{2}\right) \cap L^{1}\left(\mathbb{T} \times \mathbb{R}^{2}\right)\right),\|u\|_{H^{4} \cap L^{1}} \leq \varepsilon$ and $M(t) \leq 1$, then for small $\varepsilon>0$

(i) $\|u\|_{L^{\infty}} \leq C \varepsilon^{\frac{1}{6}}(1+t)^{-\frac{7}{12}} M^{\frac{5}{8}}(t)$.

(ii) $\|\mathcal{G}(\phi, m)\|_{L^{1}} \leq C(1+t)^{-1} M^{2}(t)$.

(iii) $\|g(\phi, m)\|_{L^{1}} \leq C(1+t)^{-1} M^{2}(t)$.

(iv) $\|G\|_{H^{1}} \leq C \varepsilon^{\frac{13}{15}}(1+t)^{-1} M(t)+C \varepsilon^{\frac{1}{6}}(1+t)^{-\frac{13}{12}} M^{\frac{5}{4}}(t)$.

(v) $\|G\|_{L^{1}} \leq C \varepsilon^{\frac{1}{3}}(1+t)^{-\frac{7}{6}} M^{\frac{4}{3}}(t)$.

Proof. It follows from Lemma 4.1 and a direct computation that

$$
\begin{aligned}
\|u\|_{L^{\infty}} & \leq\|\bar{u}\|_{L^{\infty}}+\|\tilde{u}\|_{L^{\infty}} \\
& \leq C\|\bar{u}\|_{2}^{\frac{1}{2}}\left\|D^{2} \bar{u}\right\|_{2}^{\frac{1}{2}}+C\|\tilde{u}\|_{2}^{\frac{5}{8}}\left\|D^{4} \tilde{u}\right\|_{2}^{\frac{3}{8}} \\
& \leq C\|\bar{u}\|_{2}^{\frac{1}{2}}\|D \tilde{u}\|_{2}^{\frac{1}{3}}\left\|D^{4} \bar{u}\right\|_{2}^{\frac{1}{6}}+C\|\tilde{u}\|_{2}^{\frac{5}{8}}\left\|D^{4} \tilde{u}\right\|_{2}^{\frac{3}{8}} \\
& \leq C(1+t)^{-\frac{7}{12}} M^{\frac{5}{6}}(t) \varepsilon^{\frac{1}{6}}+C(1+t)^{-\frac{5}{8}} M^{\frac{5}{8}}(t) \varepsilon^{\frac{3}{8}} \\
& \leq C \varepsilon^{\frac{1}{6}}(1+t)^{-\frac{7}{12}} M^{\frac{5}{8}}(t),
\end{aligned}
$$

which means that (i) holds.

Since

$$
\|u \cdot u\|_{L^{1}} \leq\|u\|_{L^{2}}\|u\|_{L^{2}} \leq C(1+t)^{-1} M^{2}(t)
$$

and

$$
\|u \cdot D u\|_{L^{1}} \leq\|u\|_{L^{2}}\|D u\|_{L^{2}} \leq C(1+t)^{-\frac{3}{2}} M^{2}(t),
$$

we arrive at

$$
\|\mathcal{G}(\phi, m)\|_{L^{1}} \leq C\|u\|_{H^{1}}^{2}\|u\|_{L^{2}} \leq C(1+t)^{-1} M^{2}(t)
$$

and

$$
\|g(\phi, m)\|_{L^{1}} \leq C\|u\|_{H^{1}}^{2}\|u\|_{L^{2}} \leq C(1+t)^{-1} M^{2}(t) .
$$

Thus, (ii) and (iii) are proved.

Next we show (iv). Note that

$$
\|u \cdot u\|_{L^{2}} \leq C\|u\|_{L^{4}}^{2} \leq C\|u\|_{L^{2}}^{\frac{1}{4}}\|D u\|_{L^{2}}^{\frac{3}{4}}\|u\|_{L^{2}}^{\frac{1}{4}}\|D u\|_{L^{2}}^{\frac{3}{4}}
$$


18

$$
\begin{aligned}
& \quad \leq C(1+t)^{-\frac{7}{4}} M^{2}(t) \\
& \|D(u \cdot u)\|_{L^{2}} \leq C\|D u \cdot u\|_{L^{2}} \leq C\|D u\|_{L^{2}}\|u\|_{L^{\infty}} \\
& \quad \leq C \varepsilon^{\frac{1}{6}}(1+t)^{-\frac{19}{12}} M^{\frac{13}{8}}(t) \\
& \left\|D^{2}(u \cdot u)\right\|_{L^{2}} \leq C\left\|D^{2} u \cdot u\right\|_{L^{2}}+C\|D u \cdot D u\|_{L^{2}} \leq C\left\|D^{2} u\right\|_{L^{2}}\|u\|_{L^{\infty}}+C\|D u\|_{L^{4}}^{2} \\
& \quad \leq C\|u\|_{L^{\infty}}\|D u\|_{L^{2}}^{\frac{1}{2}}\left\|D^{3} u\right\|_{L^{2}}^{\frac{1}{2}}+C\|D u\|_{L^{2}}^{\frac{5}{4}}\left\|D^{3} u\right\|_{L^{2}}^{\frac{3}{4}} \\
& \quad \leq C \varepsilon^{\frac{2}{3}}(1+t)^{-\frac{13}{12}} M^{\frac{5}{4}}(t)
\end{aligned}
$$

and

$$
\begin{aligned}
\left\|D^{3}(u \cdot u)\right\|_{L^{2}} & \leq C\|u\|_{L^{5}}\left\|D^{3} u\right\|_{L^{\frac{10}{3}}}+C\left\|D^{2} u\right\|_{L^{2}}\|D u\|_{L^{\infty}} \\
& \leq C\|u\|_{L^{2}}^{\frac{1}{10}}\|D u\|_{L^{2}}^{\frac{9}{10}}\|D u\|_{L^{2}}^{\frac{2}{15}}\left\|D^{4} u\right\|_{L^{2}}^{\frac{13}{15}}+C\|D u\|_{L^{2}}^{\frac{1}{2}}\left\|D^{3} u\right\|_{L^{2}}^{\frac{1}{2}}\|D u\|_{L^{2}}^{\frac{1}{2}}\left\|D^{4} u\right\|_{L^{2}}^{\frac{1}{2}} \\
& \leq C \varepsilon^{\frac{13}{15}}(1+t)^{-\frac{13}{12}} M \frac{17}{15}(t)+C \varepsilon(1+t)^{-1} M(t) \\
& \leq C \varepsilon^{\frac{13}{15}}(1+t)^{-1} M(t) .
\end{aligned}
$$

Thus, by (4.4)-(4.6) we have

$\left\|\operatorname{div}\left(\frac{\gamma}{\phi+\gamma} m \otimes m\right)+\nabla F(\phi)\right\|_{L^{2}} \leq C\|D(u \cdot u)\|_{L^{2}}+C\|D \phi \cdot u \cdot u\|_{L^{2}} \leq C \varepsilon^{\frac{1}{6}}(1+t)^{-\frac{19}{12}} M^{\frac{13}{8}}(t)$

and

$$
\left\|\nu_{1} \Delta\left(\frac{\phi}{\phi+\gamma} m\right)+\nu_{2} \nabla \operatorname{div}\left(\frac{\phi}{\phi+\gamma} m\right)\right\|_{L^{2}} \leq C \varepsilon^{\frac{2}{3}}(1+t)^{-\frac{13}{12}} M^{\frac{5}{4}}(t) .
$$

Since

$$
G(\phi, m)=-\operatorname{div}\left(\frac{\gamma}{\phi+\gamma} m \otimes m\right)-\nabla F(\phi)-\nu_{1} \Delta\left(\frac{\phi}{\phi+\gamma} m\right)-\nu_{2} \nabla \operatorname{div}\left(\frac{\phi}{\phi+\gamma} m\right),
$$

then substituting (4.8)-(4.9) into (4.10) yields

$$
\|G\|_{L^{2}} \leq C \varepsilon^{\frac{1}{6}}(1+t)^{-\frac{13}{12}} M^{\frac{5}{4}}(t) .
$$

Similarly, applying (4.7) and (4.11), we have

$$
\|G\|_{H^{1}} \leq C\left\|D^{3}(u \cdot u)\right\|_{L^{2}}+C\|G\|_{L^{2}} \leq C \varepsilon^{\frac{13}{15}}(1+t)^{-1} M(t)+C \varepsilon^{\frac{1}{6}}(1+t)^{-\frac{13}{12}} M^{\frac{5}{4}}(t) .
$$

Finally, we prove (v). It follows a direct computation that

$$
\begin{aligned}
\|G\|_{L^{1}} & \leq C\|D(u \cdot u)\|_{L^{1}}+C\left\|D^{2}(u \cdot u)\right\|_{L^{1}} \leq C\|u\|_{L^{2}}\|D u\|_{L^{2}}+C\|D u\|_{L^{2}}^{2}+C\left\|D^{2} u\right\|_{L^{2}}\|u\|_{L^{2}} \\
& \leq C(1+t)^{-\frac{3}{2}} M^{2}(t)+C(1+t)^{-2} M^{2}(t)+C\|D u\|_{L^{2}}^{\frac{2}{3}}\left\|D^{4} u\right\|_{L^{2}}^{\frac{1}{3}}\|u\|_{L^{2}} \\
& \leq C(1+t)^{-\frac{3}{2}} M^{2}(t)+C(1+t)^{-2} M^{2}(t)+C \varepsilon^{\frac{1}{3}}(1+t)^{-\frac{7}{6}} M^{\frac{4}{3}}(t) \\
& \leq C \varepsilon^{\frac{1}{3}}(1+t)^{-\frac{7}{6}} M^{\frac{4}{3}}(t) .
\end{aligned}
$$


For later uses, we specially list some intermediate results in the proof procedure of Lemma 4.2 as follows:

Lemma 4.3. Assuming $u \in C\left([0,+\infty), H^{4}\left(\mathbb{T} \times \mathbb{R}^{2}\right) \cap L^{1}\left(\mathbb{T} \times \mathbb{R}^{2}\right)\right),\|u\|_{H^{4} \cap L^{1}} \leq \varepsilon$ and $M(t) \leq 1$, then we have

(i) $\|u \cdot u\|_{L^{2}} \leq C(1+t)^{-\frac{7}{4}} M^{2}(t)$.

(ii) $\|D(u \cdot u)\|_{L^{2}} \leq C \varepsilon^{\frac{1}{6}}(1+t)^{-\frac{19}{12}} M^{\frac{13}{8}}(t)$.

(iii) $\left\|D^{2}(u \cdot u)\right\|_{L^{2}} \leq C \varepsilon^{\frac{2}{3}}(1+t)^{-\frac{13}{12}} M^{\frac{5}{4}}(t)$.

(iv) $\left\|D^{3}(u \cdot u)\right\|_{L^{2}} \leq C \varepsilon^{\frac{13}{15}}(1+t)^{-1} M(t)$.

Next we establish some estimates on $M[\bar{u}](t)$.

Lemma 4.4. Assuming $u \in C\left([0,+\infty), H^{4}\left(\mathbb{T} \times \mathbb{R}^{2}\right) \cap L^{1}\left(\mathbb{T} \times \mathbb{R}^{2}\right)\right),\|u\|_{H^{4} \cap L^{1}} \leq \varepsilon$ and $M(t) \leq 1$, then one has

$$
M[\bar{u}](t) \leq C \varepsilon+C \varepsilon^{\frac{1}{6}} M(t)+C M^{\frac{4}{3}}(t) .
$$

Proof. By (4.2) and Lemma 2.1, in order to show (4.12), we only need to estimate the integral

$$
\begin{aligned}
\int_{0}^{t} \overline{\mathcal{U}}(t-\tau) \bar{f}(\tau, y) d \tau & =\int_{0}^{t} \overline{\mathcal{U}}_{(0)}(t-\tau) \bar{f}(\tau, y) d \tau+\int_{0}^{t} \overline{\mathcal{U}}_{(\infty)}(t-\tau) \bar{f}(\tau, y) d \tau \\
& \triangleq J_{1}(t, y)+J_{2}(t, y) .
\end{aligned}
$$

For $J_{1}(t, y)$, by Lemma 2.1 (ii), Lemma 4.2 (ii)-(iii) and the expression of $G$, we have for $j=0,1$,

$$
\begin{aligned}
& \left\|\partial_{y}^{j} J_{1}(t, y)\right\|_{L^{2}} \leq C \int_{\frac{t}{2}}^{t}\left\|\mathcal{U}_{(0)}(t-\tau) \partial_{y}^{j+1}(\overline{\mathcal{G}}+\bar{g})\right\|_{L^{2}} d \tau+C \int_{0}^{\frac{t}{2}}\left\|\partial_{y}^{j} \mathcal{U}_{(0)}(t-\tau) \bar{G}\right\|_{L^{2}} d \tau \\
& \quad \leq C \int_{\frac{t}{2}}^{t}(1+t-\tau)^{-\frac{j+2}{2}}\|\overline{\mathcal{G}}+\bar{g}\|_{L^{1}} d \tau+C \int_{0}^{\frac{t}{2}}(1+t-\tau)^{-\frac{j+1}{2}}\|\bar{G}\|_{L^{1}} d \tau \\
& \quad \leq C(1+t)^{-1} M^{2}(t) \int_{\frac{t}{2}}^{t}(1+t-\tau)^{-\frac{j+2}{2}} d \tau+C \varepsilon^{\frac{1}{3}}(1+t)^{-\frac{j+1}{2}} M^{\frac{4}{3}}(t) \int_{0}^{\frac{t}{2}}(1+\tau)^{-\frac{7}{6}} d \tau \\
& \quad \leq C(1+t)^{-\frac{j+1}{2}} M^{\frac{4}{3}}(t) .
\end{aligned}
$$

On the other hand, we have

$$
\left\|J_{2}(t, y)\right\|_{H^{1}} \leq C M(t) \int_{0}^{t} e^{-a_{0}(t-\tau)} \varepsilon^{\frac{1}{6}}(1+\tau)^{-1} d \tau \leq C \varepsilon^{\frac{1}{6}}(1+t)^{-1} M(t) .
$$

Consequently,

$$
\|\bar{u}\|_{2} \leq C(1+t)^{-\frac{1}{2}}\left\|\bar{u}_{0}\right\|_{2}+C \varepsilon^{\frac{1}{6}}(1+t)^{-1} M(t)+(1+t)^{-\frac{1}{2}} M^{\frac{4}{3}}(t)
$$


and

$$
\|D \bar{u}\|_{2} \leq C(1+t)^{-1}\left\|D \bar{u}_{0}\right\|_{2}+C \varepsilon^{\frac{1}{6}}(1+t)^{-1} M(t)+(1+t)^{-1} M^{\frac{4}{3}}(t) .
$$

Then

$$
M[\bar{u}](t) \leq C \varepsilon+C \varepsilon^{\frac{1}{6}} M(t)+C M^{\frac{4}{3}}(t)
$$

and the proof of Lemma 4.4 is completed.

Next, we treat the difference $\tilde{u}=u-\bar{u}$. It is noted that $\tilde{u}$ satisfies the following system

$$
\left\{\begin{array}{l}
\partial_{t} \tilde{u}+L \tilde{u}=f(\phi, m)-\bar{f}(\phi, m) \triangleq \tilde{f}(t, z), \\
\tilde{u}(0, z)=\tilde{u}_{0}(z)=u_{0}(z)-\bar{u}_{0}(y) .
\end{array}\right.
$$

then by Duhamel's principle, we have

$$
\tilde{u}(t, z)=\mathcal{U}(t) \tilde{u}_{0}+\int_{0}^{t} \mathcal{U}(t-\tau) \tilde{f}(\tau, z) d \tau .
$$

Using Lemma 2.1, we see that

$$
\|\tilde{u}\|_{H^{1}} \leq C e^{-a_{0} t}\left\|\tilde{u}_{0}\right\|_{H^{1}}+C \int_{0}^{t} e^{a_{0}(t-\tau)}\|\tilde{f}(\tau, z)\|_{H^{1}} d \tau .
$$

Furthermore, we have

Lemma 4.5. Assuming $u \in C\left([0,+\infty), H^{4}\left(\mathbb{T} \times \mathbb{R}^{2}\right) \cap L^{1}\left(\mathbb{T} \times \mathbb{R}^{2}\right)\right)$, $\|u\|_{H^{4} \cap L^{1}} \leq \varepsilon$ and $M(t) \leq 1$, then

$$
(1+t)\|\tilde{u}\|_{H^{1}} \leq C\left(\varepsilon+\varepsilon^{\frac{13}{15}} M(t)+\varepsilon^{\frac{1}{6}} M^{\frac{5}{4}}(t)\right) .
$$

Proof. By Lemma 4.2, we have

$$
\begin{aligned}
\|\tilde{u}\|_{H^{1}} & \leq C e^{-a_{0} t}\left\|\tilde{u}_{0}\right\|_{H^{1}}+C \int_{0}^{t} e^{-a_{0}(t-\tau)}\|\tilde{f}(\tau, z)\|_{H^{1}} d \tau \\
& \leq C e^{-a_{0} t}\left\|\tilde{u}_{0}\right\|_{H^{1}}+C \int_{0}^{t} e^{-a_{0}(t-\tau)}\left\{\varepsilon^{\frac{13}{15}}(1+\tau)^{-1} M(\tau)+C \varepsilon^{\frac{1}{6}}(1+t)^{-\frac{13}{12}} M^{\frac{5}{4}}(\tau)\right\} d \tau \\
& \leq C e^{-a_{0} t}\left\|\tilde{u}_{0}\right\|_{H^{1}}+C \varepsilon^{\frac{13}{15}}(1+t)^{-1} M(t)+C \varepsilon^{\frac{1}{6}}(1+t)^{-\frac{13}{12}} M^{\frac{5}{4}}(t)
\end{aligned}
$$

and further

$$
(1+t)\|\tilde{u}\|_{H^{1}} \leq C\left(\varepsilon+\varepsilon^{\frac{13}{15}} M(t)+\varepsilon^{\frac{1}{6}} M^{\frac{5}{4}}(t)\right) .
$$

This completes the proof of Lemma 4.5.

Finally, we conclude the decay results of $u(t, z)$ in the section.

Proposition 4.6. For small $\varepsilon>0$, if $u_{0} \in H^{4}\left(\mathbb{T} \times \mathbb{R}^{2}\right)$ and $\left\|u_{0}\right\|_{H^{4} \cap L^{1}} \leq \varepsilon$, then we have

$$
\begin{gathered}
\left\|\partial_{z}^{k} u(t, z)\right\|_{L^{2}}=O\left(t^{-\frac{1}{2}-\frac{k}{2}}\right), \quad k=0,1, \\
\left\|\partial_{z}^{k}(u(t, z)-\bar{u}(t, y))\right\|_{L^{2}}=O\left(t^{-1}\right), \quad k=0,1,
\end{gathered}
$$


where $u$ is the solution of (1.3) and $\bar{u}$ is the solution of the system (4.3).

Proof. From Lemma 4.4-4.5, we see that

$$
M(t) \leq C\left(\varepsilon+\varepsilon^{\frac{1}{6}} M(t)+\varepsilon^{\frac{1}{6}} M^{\frac{5}{4}}(t)\right),
$$

which implies $M(t) \leq C \varepsilon$ for small $\varepsilon>0$ and further

$$
\left\|\partial_{z}^{k} u(t, z)\right\|_{L^{2}}=O\left(t^{-\frac{1}{2}-\frac{k}{2}}\right), \quad k=0,1 .
$$

In addition, by Lemma 4.5, we can easily obtain

$$
\left\|\partial_{z}^{k}(u(t, z)-\bar{u}(t, y))\right\|_{L^{2}}=O\left(t^{-1}\right), \quad k=0,1 .
$$

Thus Proposition 4.6 is proved.

\section{§5. Large-time behavior of the solution to (1.7) and the proof of Theorem 1.1}

In this section, we will mainly complete the proof of Theorem 1.1. To this end, at first we study the asymptotic behavior of the solution to (1.7). Let $\eta$ and $\bar{u}$ be the solutions of (1.7) and (4.3), respectively. We define

$$
N_{1}(t)=\sup _{0 \leq \tau \leq t}(1+\tau)\|\bar{u}(t, y)-\eta(t, y)\|_{H^{1}\left(\mathbb{R}^{2}\right)}
$$

and

$$
h=\left(\begin{array}{c}
0 \\
h_{1}
\end{array}\right)=\left(\begin{array}{c}
0 \\
-\operatorname{div}^{\prime}\left(w_{1} w^{\prime}\right) \\
-\operatorname{div}^{\prime}\left(w^{\prime} \otimes w^{\prime}\right)-\alpha \nabla^{\prime}\left(\sigma^{2}\right)
\end{array}\right) .
$$

Lemma 5.1. If $u_{0} \in H^{4}\left(\mathbb{T} \times \mathbb{R}^{2}\right)$ and $\left\|u_{0}\right\|_{H^{4} \cap L^{1}} \leq \varepsilon$, then for small $\varepsilon>0$

$$
\|\bar{u}-\eta\|_{H^{1}} \leq C(1+t)^{-1} \varepsilon .
$$

Proof. By Duhamel's principle, one has

$$
\begin{aligned}
(\bar{u}-\eta)(t, y) & =\int_{0}^{t} \overline{\mathcal{U}}(t-\tau)(\bar{f}-h)(\tau, y) d \tau \\
& =\int_{0}^{t} \overline{\mathcal{U}}_{(0)}(t-\tau)(\bar{f}-h) d \tau+\int_{0}^{t} \overline{\mathcal{U}}_{(\infty)}(t-\tau)(\bar{f}-h) d \tau \\
& =J_{1}(t, y)+J_{2}(t, y) .
\end{aligned}
$$

By Proposition 4.6 and Lemma 3.2, we see that $M(t)+M_{0}(t) \leq C \varepsilon$, where $M(t)$ and $M_{0}(t)$ are defined in $\S 4$ and Lemma 3.2 respectively. This, together with Lemma 4.2 (iv), yields

$$
\|\bar{f}-h\|_{H^{1}} \leq C\|G\|_{H^{1}}+C\left\|h_{1}\right\|_{H^{1}} \leq C \varepsilon^{2}(1+t)^{-1} .
$$


We now treat the terms $J_{1}(t, y)$ and $J_{2}(t, y)$ in the right hand side of (5.1) separately. At first, we deal with $J_{2}(t, y)$. It follows from a direct computation that

$$
\begin{aligned}
\left\|J_{2}\right\|_{H^{1}} & \leq C \int_{0}^{t} e^{-a_{0}(t-\tau)}\|\bar{f}-h\|_{H^{1}} d \tau \\
& \leq C \int_{0}^{t} e^{-a_{0}(t-\tau)} \varepsilon^{2}(1+\tau)^{-1} d \tau \\
& \leq C \varepsilon^{2}(1+t)^{-1} .
\end{aligned}
$$

Next, we deal with $J_{1}(t, y)$. It is noted that $\bar{f}$ can be written as

$$
\begin{aligned}
& \bar{f}=\left(\begin{array}{c}
0 \\
\Pi\left[-\operatorname{div}^{\prime}\left(\frac{\gamma}{\gamma+\phi} m_{1} m^{\prime}\right)\right] \\
\Pi\left[-\operatorname{div}^{\prime}\left(\frac{\gamma}{\gamma+\phi} m^{\prime} \otimes m^{\prime}\right)-\nabla^{\prime} F(\phi)\right]
\end{array}\right)+\left(\begin{array}{c}
0 \\
\Pi\left[-\nu_{1} \Delta^{\prime}\left(\frac{\phi}{\gamma+\phi} m_{1}\right)\right] \\
\Pi\left[-\nu_{1} \Delta^{\prime}\left(\frac{\phi}{\gamma+\phi} m^{\prime}\right)-\nu_{2} \nabla^{\prime} \operatorname{div}^{\prime}\left(\frac{\phi}{\gamma+\phi} m^{\prime}\right)\right]
\end{array}\right) \\
& =\bar{f}_{1}+\bar{f}_{2} .
\end{aligned}
$$

From Lemma 2.1 (i), we see that

$$
\begin{aligned}
& \left\|\partial_{y}^{j} \int_{0}^{t} \overline{\mathcal{U}}_{(0)}(t-\tau) \bar{f}_{2}(\tau, y) d \tau\right\|_{L^{2}} \leq C\left\|\partial_{y}^{j} \int_{0}^{t} \overline{\mathcal{U}}_{(0)}(t-\tau) \partial_{y}^{2}\left(\frac{\phi}{\gamma+\phi} m\right) d \tau\right\|_{L^{2}} \\
& \leq C \int_{\frac{t}{2}}^{t}(1+t-\tau)^{-\frac{3}{2}-\frac{j}{2}}\|\phi m\|_{L^{1}} d \tau+C \int_{0}^{\frac{t}{2}}(1+t-\tau)^{-1-\frac{j}{2}}\left\|\partial_{y}(\phi \cdot m)\right\|_{L^{1}} d \tau \\
& \leq C \int_{\frac{t}{2}}^{t}(1+t-\tau)^{-\frac{3}{2}-\frac{j}{2}} \varepsilon^{2}(1+\tau)^{-1} d \tau+C \int_{0}^{\frac{t}{2}}(1+t-\tau)^{-1-\frac{j}{2}}(1+t)^{-\frac{3}{2}}(\tau) d \tau \\
& \leq C \varepsilon^{2}(1+t)^{-1} .
\end{aligned}
$$

In addition, one has

$$
\begin{aligned}
\bar{f}_{1}-h_{1}= & \left(\begin{array}{c}
0 \\
-\operatorname{div}^{\prime}\left(\Pi\left[\frac{\gamma}{\gamma+\phi} m_{1} m^{\prime}-w_{1} w^{\prime}\right]\right) \\
-\operatorname{div}^{\prime}\left(\Pi\left[\frac{\gamma}{\gamma+\phi} m^{\prime} \otimes m^{\prime}-w^{\prime} \otimes w^{\prime}\right]\right)-\nabla^{\prime} \Pi\left[F(\phi)-\alpha \sigma^{2}\right]
\end{array}\right) \\
= & \left(\begin{array}{c}
0 \\
-\operatorname{div}^{\prime}\left(\Pi\left[\frac{\gamma}{\gamma+\phi} m_{1} m^{\prime}-\bar{m}_{1} \bar{m}^{\prime}\right]\right) \\
-\operatorname{div}^{\prime}\left(\Pi\left[\frac{\gamma}{\gamma+\phi} m^{\prime} \otimes m^{\prime}-\bar{m}^{\prime} \otimes \bar{m}^{\prime}\right]\right)-\nabla^{\prime} \Pi\left[F(\phi)-\alpha \bar{\sigma}^{2}\right]
\end{array}\right) \\
& +\left(\begin{array}{c}
0 \\
-\operatorname{div}^{\prime}\left(\bar{m}^{\prime} \otimes \bar{m}^{\prime}-w^{\prime} \otimes w^{\prime}\right)-\nabla^{\prime}\left(\alpha \bar{\sigma}^{2}-\alpha \sigma^{2}\right)
\end{array}\right) \\
= & R_{1}(t, y)+R_{2}(t, y) .
\end{aligned}
$$

We now analyze the first term $R_{1}(t, y)$. 
Since

$$
\frac{\gamma}{\gamma+\phi} m^{\prime} \otimes m^{\prime}-\bar{m}^{\prime} \otimes \bar{m}^{\prime}=\frac{1}{\gamma+\phi}\left(\gamma \tilde{m}^{\prime} \otimes m^{\prime}+\gamma \bar{m}^{\prime} \otimes \tilde{m}^{\prime}-\phi \bar{m}^{\prime} \otimes \bar{m}^{\prime}\right)
$$

and

$$
\begin{aligned}
& \left\|\gamma \tilde{m}^{\prime} \otimes m^{\prime}+\gamma \bar{m}^{\prime} \otimes \tilde{m}^{\prime}\right\|_{L^{1}} \leq C\left\|\tilde{m}^{\prime}\right\|_{2}\left\|m^{\prime}\right\|_{2} \leq C(1+t)^{-\frac{3}{2}} \varepsilon^{2}, \\
& \left\|\frac{1}{\gamma+\phi} \phi \bar{m}^{\prime} \otimes \bar{m}^{\prime}\right\|_{L^{1}} \leq C\|\phi\|_{L^{2}}\left\|\bar{m}^{\prime}\right\|_{L^{\infty}}\left\|\partial_{y} \bar{m}^{\prime}\right\|_{L^{2}} \leq C(1+t)^{-\frac{31}{12}} \varepsilon^{\frac{67}{24}}
\end{aligned}
$$

substituting (5.7)-(5.8) into (5.6) yields

$$
\left\|\frac{\gamma}{\gamma+\phi} m^{\prime} \otimes m^{\prime}-\bar{m}^{\prime} \otimes \bar{m}^{\prime}\right\|_{L^{1}} \leq C(1+t)^{-\frac{3}{2}} \varepsilon^{2} .
$$

On the other hand, if we denote $\alpha_{1}(\phi)=\frac{1}{\gamma^{2}} \int_{0}^{1}(1-\theta)^{2} p^{\prime \prime}\left(1+\frac{\theta \phi}{\gamma}\right) d \theta$, then

$$
F(\phi)-\alpha \bar{\phi}^{2}=\alpha_{1}(\phi)(\tilde{\phi} \phi+\bar{\phi} \tilde{\phi})+\left(\alpha_{1}(\phi)-\alpha\right) \bar{\phi}^{2} .
$$

Noting

$$
\alpha_{1}(\phi)-\alpha=\frac{1}{\gamma^{2}} \int_{0}^{1}(1-\theta)^{2} p^{\prime \prime}\left(1+\frac{\theta \phi}{\gamma}\right) d \theta-\alpha=O(\phi)
$$

then we have

$$
\left\|F(\phi)-\alpha \bar{\phi}^{2}\right\|_{L^{1}} \leq C(1+t)^{-\frac{3}{2}} \varepsilon^{2} .
$$

Combining (5.9) with (5.11) yields

$\left\|\frac{\gamma}{\gamma+\phi} m^{\prime} \otimes m^{\prime}-\bar{m}^{\prime} \otimes \bar{m}^{\prime}\right\|_{L^{1}}+\left\|\frac{\gamma}{\gamma+\phi} m_{1} m^{\prime}-\bar{m}_{1} \bar{m}^{\prime}\right\|_{L^{1}}+\left\|F(\phi)-\alpha \bar{\phi}^{2}\right\|_{L^{1}} \leq C(1+t)^{-\frac{3}{2}} \varepsilon^{2}$.

From this and the expression of $R_{1}(t, y)$, we obtain for $j=0,1$,

$$
\begin{aligned}
& \left\|\partial_{y}^{j} \int_{0}^{t} \overline{\mathcal{U}}_{(0)}(t-\tau) R_{1}(\tau, y) d \tau\right\|_{L^{2}} \\
& \leq C \int_{0}^{t}(1+t-\tau)^{-\frac{j+2}{2}}(1+\tau)^{-\frac{3}{2}} \varepsilon^{2} d \tau \leq C(1+t)^{-1} \varepsilon^{2} .
\end{aligned}
$$

Finally, we estimate $R_{2}(t, y)$. We see that for any fixed $\delta>0$

$$
\begin{aligned}
& \left\|w_{1} w^{\prime}-\bar{m}_{1} \bar{m}^{\prime}\right\|_{L^{1}} \leq\left\|w_{1}-\bar{m}_{1}\right\|_{L^{2}}\left\|\bar{w}^{\prime}\right\|_{L^{2}}+\left\|w^{\prime}-\bar{m}^{\prime}\right\|_{L^{2}}\left\|\bar{m}_{1}\right\|_{L^{2}} \leq C(1+t)^{-\frac{3}{2}} \varepsilon N_{1}(t), \\
& \left\|\sigma^{2}-\bar{\phi}^{2}\right\|_{L^{1}} \leq\|\sigma-\bar{\phi}\|_{L^{2}}\|\sigma+\bar{\phi}\|_{L^{2}} \leq C(1+t)^{-\frac{3}{2}} \varepsilon N_{1}(t), \\
& \left\|w^{\prime} \otimes w^{\prime}-\bar{m}^{\prime} \otimes \bar{m}^{\prime}\right\|_{L^{1}} \leq\left\|w^{\prime}-\bar{m}^{\prime}\right\|_{L^{2}}\left\|\bar{w}^{\prime}\right\|_{L^{2}}+\left\|w^{\prime}-\bar{m}^{\prime}\right\|_{L^{2}}\left\|\bar{m}^{\prime}\right\|_{L^{2}} \leq C(1+t)^{-\frac{3}{2}+\delta} \varepsilon N_{1}(t) .
\end{aligned}
$$

This, together with the expression of $R_{2}(t, y)$, yields

$\left\|\partial_{y}^{j} \int_{0}^{t} \overline{\mathcal{U}}_{(0)}(t-\tau) R_{2}(\tau, y) d \tau\right\|_{L^{2}}$ 


$$
\begin{aligned}
& \leq C N_{1}(t) \int_{\frac{t}{2}}^{t}(1+t-\tau)^{-1-\frac{j}{2}}(1+\tau)^{-\frac{3}{2}+\delta} \varepsilon d \tau+C N_{1}(t) \int_{0}^{\frac{t}{2}}(1+t-\tau)^{-1-\frac{j}{2}}(1+\tau)^{-\frac{3}{2}+\delta} \varepsilon d \tau \\
& \leq C(1+t)^{-1} \varepsilon N_{1}(t) .
\end{aligned}
$$

From (5.12)-(5.13), (5.5) and (5.4), we arrive at

$$
\|\bar{u}-\eta\|_{H^{1}} \leq C(1+t)^{-1} \varepsilon+C(1+t)^{-1} \varepsilon N_{1}(t)
$$

which derives for small $\varepsilon>0$

$$
N_{1}(t) \leq C \varepsilon
$$

Then we complete the proof of Lemma 5.1.

Proof of Theorem 1.1. From Remark 1.1, we know that (1.3) has a global solution $u(t, z)=$ $(\phi, m) \in C\left([0,+\infty), H^{4}\left(\mathbb{T} \times \mathbb{R}^{2}\right)\right) \cap C^{1}\left([0,+\infty), H^{2}\left(\mathbb{T} \times \mathbb{R}^{2}\right)\right)$. In addition, (1.4) comes from (4.13) directly, and (1.5) comes from the interpolation between (1.4) and $\|u\|_{H^{4}} \leq C \varepsilon$, and (1.6) is yielded by combining (4.14) and Lemma 5.1.

\section{$\S$ 6. The proofs of Theorem 1.2 and Theorem 1.3}

In this section, at first we focus on the proof of Theorem 1.2. To this end, we will derive some decay properties of the solution to (1.3) for $u_{0}(z) \in H^{4}\left(\mathbb{T}^{2} \times \mathbb{R}\right)$ as in $\S 4$. Define

$$
\Pi[g](y)=\bar{g}(y)=\frac{1}{(2 \pi)^{2}} \int_{\mathbb{T}^{2}} g\left(x_{1}, x_{2}, y\right) d x_{1} d x_{2} .
$$

Then as in (4.3), the mean value $\bar{u}(t, y)$ of the solution $u(t, z)$ to (1.3) over $\mathbb{T}^{2}$ satisfies a similar nonlinear partial differential system. Let $\tilde{u}(t, z)=u(t, z)-\bar{u}(t, y)$ and

$$
M_{1}(t)=M_{1}[u](t)=\sup _{0 \leq \tau \leq t}\left\{(1+\tau)^{\frac{1}{4}}\|u(\tau, y)\|_{L^{2}\left(\mathbb{T}^{2} \times \mathbb{R}\right)}+(1+\tau)^{\frac{3}{4}}\|\nabla u(\tau, y)\|_{L^{2}\left(\mathbb{T}^{2} \times \mathbb{R}\right)}\right\} .
$$

By the analogous proof in Lemma 4.2, we can obtain

Lemma 6.1. Assuming $u \in C\left([0,+\infty), H^{4}\left(\mathbb{T}^{2} \times \mathbb{R}\right) \cap L^{1}\left(\mathbb{T}^{2} \times \mathbb{R}\right)\right),\|u\|_{H^{4} \cap L^{1}} \leq \varepsilon$ and $M_{1}(t) \leq 1$, then we have for small $\varepsilon>0$

(i) $\|u\|_{L^{\infty}} \leq C(1+t)^{-\frac{1}{2}} M_{1}(t)+C \varepsilon^{\frac{1}{2}}(1+t)^{-\frac{3}{8}} M_{1}^{\frac{1}{2}}(t)$.

(ii) $\|\mathcal{G}(\phi, m)\|_{L^{1}} \leq C(1+t)^{-\frac{1}{2}} M_{1}^{2}(t)$.

(iii) $\|g(\phi, m)\|_{L^{1}} \leq C(1+t)^{-\frac{1}{2}} M_{1}^{2}(t)$.

(iv) $\|G\|_{H^{1}} \leq C(1+t)^{-\frac{5}{4}} M_{1}^{2}(t)+C \varepsilon^{\frac{1}{2}}(1+t)^{-\frac{3}{4}} M_{1}(t)$.

Where the definitions of $\mathcal{G}(\phi, m), g(\phi, m)$ and $G$ have been given at the beginning of $\S 4$

Based on Lemma 6.1, by applying Remark 3.1 and Lemma 4.1 (ii), as in the proofs of Lemma 4.4 and Lemma 4.5, we have

Lemma 6.2. Assuming $u \in C\left([0,+\infty), H^{4}\left(\mathbb{T}^{2} \times \mathbb{R}\right) \cap L^{1}\left(\mathbb{T}^{2} \times \mathbb{R}\right)\right),\|u\|_{H^{4} \cap L^{1}} \leq \varepsilon$ and $M_{1}(t) \leq 1$, then

$$
\text { (i) } M_{1}[\bar{u}](t) \leq C\left(\varepsilon+\varepsilon M_{1}(t)+M_{1}^{2}(t)\right) \text {. }
$$




$$
\text { (ii) } M_{1}[\tilde{u}](t) \leq C\left(\varepsilon+\varepsilon^{\frac{1}{2}} M_{1}(t)+M_{1}^{2}(t)\right) \text {. }
$$

From Lemma 6.2, we can easily derive the following results similar to Proposition 4.6:

Proposition 6.3. For small $\varepsilon>0$, if $u_{0} \in H^{4}\left(\mathbb{T}^{2} \times \mathbb{R}\right)$ and $\left\|u_{0}\right\|_{H^{4} \cap L^{1}} \leq \varepsilon$, then we have

$$
\begin{gathered}
\left\|\partial_{z}^{k} u(t, z)\right\|_{L^{2}}=O\left(t^{-\frac{1}{4}-\frac{k}{2}}\right), \quad k=0,1, \\
\left\|\partial_{z}^{k}(u(t, z)-\bar{u}(t, y))\right\|_{L^{2}}=O\left(t^{-\frac{3}{4}}\right), \quad k=0,1,
\end{gathered}
$$

where $u$ is the solution of (1.3) and $\bar{u}$ is the mean value of $u$ over $\mathbb{T}^{2}$.

In addition, as in Lemma 5.1, one has

Lemma 6.4. If $u_{0} \in H^{4}\left(\mathbb{T}^{2} \times \mathbb{R}\right)$ and $\left\|u_{0}\right\|_{H^{4} \cap L^{1}} \leq \varepsilon$, then for small $\varepsilon>0$ and any fixed constant $\delta>0$

$$
\|\bar{u}-\eta\|_{H^{1}} \leq C(1+t)^{-\frac{3}{4}+\delta} \varepsilon .
$$

Proof of Theorem 1.2. Since we have established the crucial Proposition 6.3 and Lemma 6.4, the proof of Theorem 1.2 is completely analogous to that for Theorem 1.2, we omit the details here.

Finally, we prove Theorem 1.3.

Proof of Theorem 1.3. By (2.7), it is easy to see that the meanvalues $\bar{u}$ of $u(t, z)$ over $\mathbb{T}^{3}$ are constants, namely, $(\bar{\phi}, \bar{m}) \equiv\left(\bar{\phi}_{0}, \bar{m}_{0}\right)$. Denote $\tilde{u}=u-\bar{u}$, then it follows from (1.3) that

$$
\left\{\begin{aligned}
\partial_{t} \tilde{\phi}+ & \gamma \operatorname{div} \tilde{m}=0, \\
\partial_{t} \tilde{m}+ & \left(-\frac{\gamma}{(\bar{\phi}+\gamma)^{2}} \bar{m}(\bar{m} \cdot \nabla)+\frac{c}{\gamma} \nabla+\frac{\nu_{1} \gamma}{(\bar{\phi}+\gamma)^{2}} \bar{m} \Delta+\frac{\nu_{2} \gamma}{(\bar{\phi}+\gamma)^{2}}(\bar{m} \cdot \nabla) \nabla\right) \tilde{\phi} \\
+ & \left(\frac{\gamma}{\bar{\phi}+\gamma} \bar{m} \operatorname{div}+\frac{\gamma}{\bar{\phi}+\gamma} \bar{m} \cdot \nabla-\frac{\gamma}{\bar{\phi}+\gamma}\left(\nu_{1} \Delta+\nu_{2} \nabla \operatorname{div}\right)\right) \tilde{m} \\
& =\nabla\left[\tilde{u}^{2} g_{1}(\bar{u}, \tilde{u})\right]+\nabla^{2}\left[\tilde{u}^{2} g_{2}(\bar{u}, \tilde{u})\right],
\end{aligned}\right.
$$

where $c=p^{\prime}\left(\frac{\bar{\phi}+\gamma}{\gamma}\right)$, and $g_{1}, g_{2}$ are smooth functions on their argumants.

If we denote

$A=$

$\left(\begin{array}{c}0 \\ -\frac{\gamma}{(\bar{\phi}+\gamma)^{2}} \bar{m}(\bar{m} \cdot \nabla)+\frac{c}{\gamma} \nabla+\frac{\nu_{1} \gamma}{(\bar{\phi}+\gamma)^{2}} \bar{m} \Delta+\frac{\nu_{2} \gamma}{(\bar{\phi}+\gamma)^{2}}(\bar{m} \cdot \nabla) \nabla \\ \frac{\gamma}{\bar{\phi}+\gamma}\left(\bar{m} \operatorname{div}+\bar{m} \nabla-\left(\nu_{1} \Delta+\nu_{2} \nabla \operatorname{div}\right)\right)\end{array}\right)$,

then the linearized system of (6.1) can be written as

$$
\left\{\begin{array}{l}
\partial_{t} v+A v=0 \\
v(0, z)=\tilde{u}_{0}(z)=u_{0}(z)-\bar{u}_{0}
\end{array}\right.
$$


Taking the Fourier series expansion for the matrix $A$, we get

$\hat{A}(k)=$

$$
\left(\begin{array}{cc}
0 & i \gamma k^{T} \\
-\frac{i \gamma}{(\phi+\gamma)^{2}}(\bar{m} \cdot k) \bar{m}+\frac{i c}{\gamma} k-\frac{\nu_{1} \gamma|k|^{2}}{(\phi+\gamma)^{2}} \bar{m}-\frac{\nu_{2} \gamma}{(\phi+\gamma)^{2}}(\bar{m} \cdot k) k & \frac{i \gamma}{\phi+\gamma} \bar{m}\left(k^{T}+k\right)+\frac{\gamma}{\phi+\gamma}\left(\nu_{1}|k|^{2}+\nu_{2} k k^{T}\right)
\end{array}\right),
$$

where $k \in \mathbb{Z}^{3}$.

It is easy to know that the eigenvalues of $\hat{A}(k)$ are

$$
\lambda_{1}=\lambda_{2}=\frac{\gamma}{\bar{\phi}+\gamma}\left(\nu_{1}|k|^{2}+i \bar{m} \cdot k\right), \lambda_{ \pm}=\frac{\frac{\gamma}{\phi+\gamma}\left(\left(\nu_{1}+\nu_{2}\right)|k|^{2}+2 i \bar{m} \cdot k\right) \pm \sqrt{\frac{\gamma^{2}\left(\nu_{1}+\nu_{2}\right)^{2}|k|^{4}}{(\phi+\gamma)^{2}}-4 c|k|^{2}}}{2} .
$$

Consequently, for small $\left(\bar{\phi}_{0}, \bar{u}_{0}\right)$ and $k \neq 0$, there exists a positive constant $a_{0}$ such that $\Re e \lambda_{\kappa} \geq$ $a_{0}$ for $\kappa=1,2, \pm$, and we can obtain

$$
\|v(t, z)\|_{H^{1}} \leq C e^{-a_{0} t}\left\|\tilde{u}_{0}\right\|_{H^{1}} .
$$

Denote $M_{2}(t)=\sup _{0 \leq \tau \leq t} e^{a_{0} t}\|\tilde{u}\|_{H^{1}}$. Noting that $\|u\|_{H^{4}} \leq \varepsilon$ and further $\|\tilde{u}\|_{H^{4}} \leq \varepsilon$ hold, then we can arrive at

$$
\begin{aligned}
\left\|\nabla\left[\tilde{u}^{2} g_{1}(\bar{u}, \tilde{u})\right]\right\|_{H^{1}} & \leq C\|\tilde{u} D \tilde{u}\|_{L^{2}}+C\left\|(D \tilde{u})^{2}\right\|_{L^{2}}+C\left\|\tilde{u} D^{2} \tilde{u}\right\|_{L^{2}} \\
& \leq C\|\tilde{u}\|_{L^{6}}\|D \tilde{u}\|_{L^{3}}+C\|D \tilde{u}\|_{L^{4}}^{2}+C\|\tilde{u}\|_{L^{6}}\left\|D^{2} \tilde{u}\right\|_{L^{3}} \\
& \leq C \varepsilon^{\frac{1}{6}}\|\tilde{u}\|_{H^{1}}^{\frac{3}{2}}
\end{aligned}
$$

and

$$
\begin{aligned}
\left\|\nabla^{2}\left[\tilde{u}^{2} g_{2}(\bar{u}, \tilde{u})\right]\right\|_{H^{1}} & \leq C\left\|(D \tilde{u})^{2}\right\|_{L^{2}}+C\left\|(D \tilde{u})^{3}\right\|_{L^{2}}+C\left\|D \tilde{u} D^{2} \tilde{u}\right\|_{L^{2}}+C\left\|\tilde{u} D^{3} \tilde{u}\right\|_{L^{2}} \\
& \leq C\|D \tilde{u}\|_{L^{4}}^{2}+C\|D \tilde{u}\|_{L^{6}}^{3}+C\|D \tilde{u}\|_{L^{3}}\left\|D^{2} \tilde{u}\right\|_{L^{6}}+C\|\tilde{u}\|_{L^{6}}\left\|D^{3} \tilde{u}\right\|_{L^{3}} \\
& \leq C \varepsilon^{\frac{1}{2}}\|\tilde{u}\|_{H^{1}}^{\frac{7}{6}} .
\end{aligned}
$$

Hence we have from (6.1) and (6.3)

$$
\begin{aligned}
\|\tilde{u}\|_{H^{1}} \leq & \|v\|_{H^{1}}+\int_{0}^{t}\left\|e^{-(t-\tau) A}\left(0, \nabla\left[\tilde{u}^{2} g_{1}(\bar{u}, \tilde{u})\right]+\nabla^{2}\left[\tilde{u}^{2} g_{2}(\bar{u}, \tilde{u})\right]\right)(\tau, y)\right\|_{H^{1}} d \tau \\
& \leq C e^{-a_{0} t}\left\|u_{0}\right\|_{H^{1}}+\varepsilon^{\frac{1}{6}} \int_{0}^{t} e^{-a_{0}(t-\tau)}\|\tilde{u}(\tau, y)\|_{H^{1}}^{\frac{7}{6}} d \tau \\
& \leq C e^{-a_{0} t}\left\|u_{0}\right\|_{H^{1}}+\varepsilon^{\frac{1}{6}} e^{-a_{0} t} M_{2}^{\frac{7}{6}}(t) \int_{0}^{t} e^{-\frac{1}{6} a_{0} \tau} d \tau \\
& \leq C e^{-a_{0} t} \varepsilon+\varepsilon^{\frac{1}{6}} e^{-a_{0} t} M_{2}^{\frac{7}{6}}(t)
\end{aligned}
$$

and further obtain

$$
M_{2}(t) \leq C \epsilon+C \epsilon^{\frac{1}{6}} M_{2}^{\frac{7}{6}}(t) .
$$

This derives that $M_{2}(t) \leq C \varepsilon$ and $\|\tilde{u}(t, y)\|_{H^{1}}$ decays exponentially with respect to the time $t$. Therefore, we complete the proof of Theorem 1.3. 


\section{References}

[1] R. A. Adams, Sobolev spaces. Pure and Applied Mathematics, Vol. 65. Academic Press, New York-London, 1975.

[2] Duan Renjun, Ma Hongfang Global existence and convergence rates for the 3-D compressible Navier-Stokes equations without heat conductivity, Indiana Univ. Math. J. 57, no. 5, 2299-2319 (2008).

[3] Duan Renjun, Liu Hongxia, S. Ukai, Yang Tong, Optimal $L^{p}-L^{q}$ convergence rates for the compressible Navier-Stokes equations with potential force, J. Differential Equations 238, no. 1, 220-233 (2007).

[4] D. Hoff, K. Zumbrun, Multi-dimensional diffusion waves for the Navier-Stokes equations of compressible flow, Indiana Univ. Math. J. 44, no. 2, 603-676 (1995).

[5] D. Hoff, K. Zumbrun, Poinwise decay estimates for multidimensional Navier-Stokes diffusion waves, Z. Angew. Math. Phys. 48, no. 4, 597-614 (1997).

[6] Y. Ishihara, Y. Kagei, Large time behavior of the semigroup on $L^{p}$ spaces associated with the linearized compressible Navier-Stokes equation in a cylindrical domain, J. Differential Equations. 248, 252-286 (2010).

[7] Y. Kagei, Large time behavior of solutions to the compressible Navier-Stokes equation in an infinite layer, Hiroshima Math. J. 38, no. 1, 95-124 (2008).

[8] Y. Kagei, Asymptotic behavior of solutions to the compressible Navier-Stokes equation around a parallel flow, Arch. Ration. Mech. Anal. 205, no. 2, 585-650 (2012).

[9] Y. Kagei, T. Nukumizu, Asymptotic behavior of solutions to the compressible NavierStokes equation in a cylindrical domain, Osaka J. Math. 4, 987-1026 (2008).

[10] T. Kobayashi, Some estimates of solutions for the equations of motion of compressible viscous fluid in the three-dimensional exterior domain, J. Differential Equations 184, 587619 (2002).

[11] Li Hailiang, Zhang Ting, Large time behavior of isentropic Compressible Navier-Stokes system in $\mathbb{R}^{3}$, Math. Methods Appl. Sci. 34, no. 6, 670-682 (2011).

[12] Liu Tai-Ping, Wang Weike, The pointwise estimates of diffusion wave for the NavierStokes systems in odd multi-dimensions, Comm. Math. Phys. 196, no. 1, 145-173 (1998).

[13] A. Matsumura, T. Nishida, The initial value problem for the equation of motion of viscous and heat-conductive gases, J. Math. Kyoto Univ. 20, no. 1, 67-104 (1980).

[14] A. Matsumura, T. Nishida, Initial boudary value problems for the equations of motion of general fluids, Comput. Methods Appl. Sci. Eng. V, 389-406 (1982). 
[15] A. Matsumura, T. Nishida, Initial boudary value problems for the equations of motion of compressible viscous and heat conductive fluids, Commun. Math. Phys. 89, 445-464 (1983).

[16] A. Tani, On the first initial-boundary value problem of compressible viscous fluid motion, Publ. RIMS. Kyoto Univ. 13, 193-253 (1977). 